\title{
Die „TraSy-Methode“ - ein Vorgehen für die transdisziplinäre Entwicklung soziotechnischer Systeme
}

\author{
Laura Gebhardt \& Alexandra König
}

\section{$1 \quad$ Einleitung}

Der Ansatz, Nutzer(innen) ${ }^{1}$ nicht nur als passive Konsument(inn)en zu sehen, sondern sie als Mitgestalter(innen) aktiv in die Entwicklung von Produkten und Lösungsstrategien zu involvieren (Mayas et al. 2012; Pruitt und Adlin 2006), wird in der Industrie bereits seit einiger Zeit verfolgt. Auch Städte und Kommunen haben erkannt, welche Vorteile es bringt, Bürger(innen) bei der Generierung neuer Ideen sowie in Planungs- und Entwicklungsprozessen zu beteiligen (Gebhardt et al. 2014; Selle 2013). Statt Wissen über Verhaltensweisen und Präferenzen von Nutzer(inne)n zu generieren und dieses danach in die Praxis zu überführen, werden Problemlösungen gemeinsam mit der Zivilgesellschaft ${ }^{2}$ vor Ort entwickelt, wie beispielweise auf der Plattform Bremenbewegen.de ${ }^{3}$. Bei den meisten dieser Ansätze bleibt jedoch unklar, wie Wissen über die Bedürfnisse unterschiedlicher Nutzergruppen (bzw. Nutzertypen) ${ }^{4}$ generiert, in Anforderungen an zu entwickelnde Systeme übersetzt und in die Lebenswelt der Betroffenen überführt wird. Unter ,System“ wird im Folgenden ,ein Komplex von Elementen, die miteinander verbunden und voneinander abhängig sind und insofern eine strukturierte Ganzheit bilden“, verstanden (Hügli und Lübcke 1991, S. 561). ,So-

1 Als Nutzer(innen) werden Personen bezeichnet, die ein Produkt oder eine Dienstleistung in Anspruch nehmen, da sie sich davon einen Nutzen versprechen.

2 Der Begriff Zivilgesellschaft benennt den Teil der außerwissenschaftlichen Gesellschaft, der nicht durch staatliche Organe organisiert wird. Somit dient der Begriff insbesondere als Abgrenzung zum staatlichen System und zum Wissenschaftssystem (Defila und Di Giulio 2018b).

3 Siehe die Webseite http://www.bremenbewegen.de (zugegriffen am 01.03.2019).

4 Nutzergruppen umfassen jeweils mehrere Nutzer(innen), die sich bezüglich forschungsrelevanter Merkmale untereinander ähnlich sind. Gleichzeitig unterscheiden sie sich dadurch von anderen Nutzer(inne)n, die nicht dieser Gruppe angehören. Die Begriffe Nutzergruppen und Nutzertypen werden im Folgenden synonym verwendet. 
ziotechnische Systeme' sind Systeme, die sowohl technische als auch nicht-technische Elemente umfassen (Büscher und Schippl 2013). Die technischen und nicht-technischen (sozialen) Elemente sind in soziotechnischen Systemen eng verknüpft (Lösch 2012). Soziotechnische Systeme berühren gesellschaftsdynamische Prozesse und sind deshalb eng mit der Transformationsforschung verbunden (Büscher und Schippl 2013). Sie können beispielsweise ein Schul- oder ein Verkehrssystem $^{5}$ sein. So umfasst ein Verkehrssystem technische Elemente wie die Verkehrsinfrastruktur mit Verkehrsmitteln und Leitsystemen für deren Koordinierung. Aber auch nicht-technische Elemente, wie Verkehrsteilnehmer(innen), die den Verkehr verursachen, sind Teil des Verkehrssystems, ebenso wie die Einrichtungen, die den Transportprozess organisieren.

Methodische Ansätze zur Erhebung von Nutzeranforderungen an neue Systeme, die davon ausgehen, dass sich Nutzer(innen) ihrer Ziele, Bedürfnisse und Anforderungen an neue soziotechnische Systeme bewusst sind und diese explizit äußern können, greifen häufig zu kurz (Brandies et al. 2017). Stattdessen müssen methodische Herangehensweisen, die zum Ziel haben, Bedürfnisse von zukünftigen Nutzer(innen) eines Systems zu identifizieren und zu beschreiben, beachten, dass diese den Nutzer(inne)n häufig selbst nicht bewusst oder leicht zugänglich sind (Achtziger und Gollwitzer 2009) und dass diese auch häufig nicht direkt beobachtbar sind (Sanders 2003). Dementsprechend sind jene Vorgehen vielversprechend, die durch den Einsatz tiefgehender und breitgefächerter Methoden, wie beispielsweise der Methode des Lauten Denkens (van Someren et al. 1994) oder der Methode der teilnehmenden Beobachtung (Lüders 2003), die eher impliziten Denkweisen und Bedürfnisse der zukünftigen Nutzer(innen) zutage bringen. Diese Methoden erlauben es den Forschenden, in die Lebenswelt der Befragten einzudringen. Wichtige Voraussetzung für den Erfolg der Identifizierung impliziter Denkweisen und Bedürfnisse der Nutzer(innen) sind dabei die Offenheit der Teilnehmer(innen) gegenüber den genannten Methoden und ihre Bereitschaft, eigene Gedanken und Einstellungen mitzuteilen.

Im vorliegenden Beitrag wird eine Methode vorgestellt, die dazu dient, Bedürfnisse heterogener Nutzergruppen eines zu entwickelnden soziotechnischen Systems systematisch zu erheben und die erhobenen Anforderungen anschließend in die konkrete Ausgestaltung dieses Systems umzusetzen. Es wird eine Methode zur transdisziplinären Entwicklung soziotechnischer Systeme vorgestellt und reflektiert. Die ,TraSy-Methode‘ (TRAnsdisziplinäre Entwicklung soziotechnischer SYsteme) wurde im Rahmen des „Reallabors Schorndorf: Zukunftsweisender Öffentlicher Verkehr - Bürgerorientierte Optimierung der Leistungsfähigkeit,

5 In diesem Beitrag wird nicht der Anspruch erhoben, den Begriff System im Sinne der soziologischen Systemtheorie (Luhmann 1984, 1997) zu verstehen und zu operationalisieren. 
Effizienz und Attraktivität im Nahverkehr“ (Reallabor Schorndorf; s. auch Steckbrief im Anhang zu diesem Buch) entwickelt und angewendet. Die TraSy-Methode wird im Folgenden vorgestellt und am Beispiel des Reallabors Schorndorf illustriert. Ziel dieses Projekts war die Entwicklung eines bedarfsgerechten und nachhaltigen Mobilitätskonzeptes für die Stadt Schorndorf (Klötzke et al. 2018). Zentral war hierbei die Frage, welche Anforderungen die Bürger(innen) der Stadt Schorndorf an das Unterwegssein stellen und wie die zukünftigen Nutzer(innen) bei der Entwicklung und Erprobung einer Mobilitätsinnovation auf Augenhöhe involviert werden können. Das neue Verkehrssystem sollte dabei keine Standalone-Lösung darstellen, sondern in das bestehende System integriert werden (König et al. 2018).

Die TraSy-Methode ist an der Schnittstelle der drei Wissensarten (System-, Zielund Transformationswissen) nach CASS und ProClim- (1997) zu verorten. Sie hat zum Ziel, durch die Analyse der aktuellen Handlungspraktiken der Nutzer(innen) Systemwissen sowie durch die Entwicklung und Erprobung eines zukünftigen soziotechnischen Systems Zielwissen zu generieren. Dabei wird ein Transformationsprozess angestoßen, bei dem Transformationswissen erzeugt wird.

Im Folgenden wird die TraSy-Methode zuerst anhand von Fragen, die sich an der Methode Interessierte stellen könnten, generell eingeführt. Anschließend wird das Vorgehen der TraSy-Methode schrittweise erklärt, um die Anwendung der TraSyMethode zu ermöglichen.

\subsection{Welche Ergebnisse können mit der TraSy-Methode erzielt werden?}

Mithilfe der TraSy-Methode können konkrete Systeme und Wissen über deren Einbettung in einem konkreten gesellschaftlichen Kontext entwickelt werden. Im Falle des Reallabors Schorndorf war dies ein appbasiertes Rufbus-System, das im Kontext des bereits bestehenden Nahverkehrsangebots der Stadt Schorndorf entwickelt wurde. Zentrales Ergebnis nach der Durchführung der TraSy-Methode soll ein System sein, das am Markt bzw. in der Gesellschaft bestehen kann, also über den Prototypenstatus hinausgeht. Ergebnisse, die während des Prozesses der Methodendurchführung entstehen und sich im Laufe des Vorgehens weiterentwickeln, sind: 1) die Beschreibung von Nutzertypen, 2) konkrete Nutzungsszenarien, 3) eine Liste mit Anforderungen unterschiedlicher Nutzertypen an das System, 4) Konzeptentwürfe des zu entwickelnden Systems und 5) Prototypen. Das Vorgehen der TraSy-Methode wird geleitet und strukturiert von zwei zentralen Arbeitsdokumenten: 1) ein Leitplankendokument, das Fragen und Antworten in Bezug auf die konkrete Ausgestaltung des Systems umfasst, und 2) ein Spezifikationsheft, das konkrete Anforderungen an das System zusammenfasst (für eine detaillierte Beschreibung von Leitplankendokument und Spezifikationsheft, s. Kapitel 2). 


\subsection{Für welche Themenbereiche ist die TraSy-Methode geeignet?}

Zwar wurde die TraSy-Methode im Bereich der Mobilitätsforschung entwickelt, jedoch kann sie in zahlreichen weiteren Bereichen angewendet werden, in denen soziotechnische Systeme entwickelt werden sollen. Denkbar ist beispielsweise die Anwendung der TraSy-Methode in Bereichen wie Infrastruktur und Städtebau (z. B. Bau eines neuen Kindergartens), Energieerzeugung (z. B. Bau einer Windkraftanlage) oder Bildung (z. B. Entwicklung einer Online-Lernplattform).

Besonders geeignet ist die Anwendung dieser Methode bei Themenbereichen, bei denen es darum geht, Anforderungen unterschiedlicher Nutzer(innen) einer heterogenen Bevölkerung an ein neues soziotechnisches System zu explorieren. $\mathrm{Zu}-$ dem ist die Methode dann besonders geeignet, wenn das notwendige Wissen für die Beschreibung der Nutzeranforderungen über verschiedene Praxisakteure ${ }^{6}$ verteilt ist. Durch einen nutzertyporientierten Ansatz über alle Entwicklungsschritte hinweg kann der Heterogenität der Bevölkerung Rechnung getragen werden. Die TraSy-Methode kann insbesondere dann eine geeignete Methode für ein Forschungsprojekt darstellen, wenn folgende Kriterien gegeben sind:

- Nutzeranforderungen an ein noch nicht existierendes soziotechnisches System sollen identifiziert, beschrieben und validiert werden.

- Das Projektthema berührt den Alltag und die täglichen Praktiken einer heterogenen Nutzergruppe.

- Neben direkten Nutzer(inne)n werden von der Entwicklung auch indirekte Nutzer(innen), wie zum Beispiel Anwohner(innen), betroffen sein.

- Das Thema berührt die Bedürfnisse und Anforderungen diverser Interessengruppen, die teilweise im Gegensatz zueinander stehen können.

- Das Thema erfordert Entscheidungen, die langfristige Effekte auf gesellschaftlich relevante Lebensbereiche haben und gesellschaftspolitische Konflikte bewirken können.

6 Als ,Praxisakteure“ werden hier Akteure aus der Praxis bezeichnet, die punktuell am Projekt beteiligt sind, die also nicht fester Bestandteil des Projektteams sind. Die Praxisakteure können aus der Zivilgesellschaft, dem Staat, Verbänden und Verbünden oder der Wirtschaft stammen. 


\subsection{Was unterscheidet die TraSy-Methode von anderen partizipativen Methoden?}

Das Vorgehen bei der TraSy-Methode zeichnet sich v. a. durch den Einsatz qualitativ-partizipativer Dialog-Methoden aus, die einen „kommunikativen Raum“ (Bergold und Thomas 2010, S. 335) für die Wissensgenerierung schaffen sollen.

Der umfassende Partizipationsansatz unterscheidet die TraSy-Methode von Methoden der partizipativen Technologiefolgenabschätzung (pTA), die vorsieht, Betroffene und Interessierte an soziotechnischen und politischen Entscheidungsprozessen zu beteiligen, um bei politischen Entscheidungen möglichst zahlreiche Aspekte eines Problems zu berücksichtigen (Joss und Bellucci 2002). Der Fokus der pTA liegt damit auf der Bewertung von technischen Entwicklungen, um Entscheidungen zu unterstützen (Hennen 2012), während die TraSy-Methode die Entwicklung eines neuen Systems gemeinsam mit den zukünftigen Nutzer(inne)n zum Ziel hat. Anders als bei der pTA arbeiten bei der TraSy-Methode Praxisakteure (insbes. Praxispartner) ${ }^{7}$ und Wissenschaftler(innen) ${ }^{8}$ gemeinsam an Aufgaben. Somit wird insbesondere den Praxispartnern aktive Mitbestimmung eingeräumt, d. h., die Deutungshoheit liegt bei den Wissenschaftler(inne)n und den Praxispartnern.

Die TraSy-Methode weist auch eine Ähnlichkeit mit den Ansätzen der Partizipativen Technikentwicklung auf (Kucharski und Merkel 2018). Gemeinsamkeiten zwischen den Ansätzen der Partizipativen Technikentwicklung und der TraSyMethode sind im iterativen Vorgehen zu sehen sowie im symmetrischen Verhältnis zwischen den Wissenschaftler(inne)n und den zukünftigen Nutzer(inne)n, die als gleichberechtigte Forschungssubjekte und nicht als Untersuchungsobjekte angesehen werden (Bergold und Thomas 2012; Müller et al. 2014). Die TraSyMethode geht jedoch über die Beteiligung von Nutzer(inne)n in der Designphase hinaus und beteiligt sie auch in den weiteren Phasen der Systementwicklung.

Die TraSy-Methode weist schließlich Aspekte des Design Thinkings auf, da sie ebenfalls auf der Annahme basiert, dass alltägliche Probleme erfolgreicher gelöst werden können, wenn die Bedürfnisse und Motive der zukünftigen Nutzer(innen) berücksichtigt werden, indem diese in einen iterativen Entwicklungsprozess einfließen (Plattner et al. 2009). Die TraSy-Methode verwendet jedoch nur Teilaspekte des Design Thinking-Ansatzes, wie beispielsweise den iterativen Charak-

7 ,Praxispartner' sind gleichberechtigte Mitglieder eines Projektteams, die in unterschiedlicher Intensität am Projekt partizipieren, jedoch immer Entscheidungsgewalt haben und kontinuierlich im Projekt mitwirken.

8 Als ,Wissenschaftler(innen)' werden Personen bezeichnet, die dem Wissenschaftssystem angehören und forschend im Projekt involviert sind (Defila und Di Giulio 2018b). 
ter der Konzeptentwicklung. Die TraSy-Methode ist im Vergleich zu einem Design Thinking-Prozess aufwendiger, aufgrund der umfangreichen Analysen von Daten aus Primär- und Sekundärquellen.

\subsection{Wer wird in der TraSy-Methode beteiligt und welche Kompetenzen der Beteiligten sind erforderlich?}

Im Sinne der transdisziplinären Forschung entspricht die TraSy-Methode der Position, Forschung mit Menschen zu betreiben, nicht über oder für Menschen (Bergold und Thomas 2010, S. 333). Deshalb werden die Nutzer(innen) in allen Teilschritten der TraSy-Methode als aktiv Partizipierende und Expert(inn)en ihrer Lebenswelt einbezogen. Dabei ist es wichtig, zu beachten, Unterschiede zwischen Wissenschaftler(inne)n und Praxisakteuren nicht zu verwischen und Praxisakteure nicht $\mathrm{zu}$ Wissenschaftler(inne)n zu machen, sondern in ihrer Rolle als Expert(inn)en der eigenen Lebenswelt zu belassen (Defila und Di Giulio 2018a).

Das Projektteam sollte aus Wissenschaftler(inne)n und Praxispartnern bestehen. Praxispartner sind Akteure, die durch ihr Praxiswissen einen wichtigen Beitrag zur Systementwicklung beitragen, da sie den Anwendungskontext überaus gut kennen. Weiterhin sind Praxispartner Vertreter bestimmter Nutzergruppen wie beispielsweise eines Seniorenverbands. Die TraSy-Methode sieht vor, dass verschiedene wissenschaftliche Disziplinen gemeinsam an der Entwicklung des neuen Systems arbeiten. Je nach thematischem Schwerpunkt des zu entwickelnden Systems werden neben Sozial- und Verhaltenswissenschaftler(inne)n auch Informatiker(innen), Ingenieur(inn)e(n) und Designer(innen) beteiligt.

Empfehlenswert ist, wenn sowohl die wissenschaftlichen als auch die außerwissenschaftlichen Teammitglieder (Praxispartner) eine Vorstellung der jeweiligen Kompetenzen und Arbeitsbereiche der anderen Teammitglieder besitzen. Falls dies nicht der Fall sein sollte, also beispielsweise die außerwissenschaftlichen Teammitglieder noch nicht in Forschungsprojekten mit Wissenschaftler(inne)n gearbeitet haben, bietet es sich an, zu Beginn ein längeres Treffen innerhalb des Projektteams zu organisieren, mit dem Ziel, die Kompetenzen und Rollen innerhalb des Projektteams zu bestimmen und die Erwartungen an die Zusammenarbeit zu diskutieren. Eine weitere Herausforderung, die sich bei der Zusammenarbeit von Forscher(inne)n und Praxispartnern ergeben kann, ist die Verständigung auf eine gemeinsame Sprache. So ist es zum Beispiel von hoher Bedeutung, dass alle Beteiligten dasselbe unter einem Begriff wie beispielsweise „System“ verstehen. Diese Aspekte sind auch in der Interaktion mit den Praxisakteuren außerhalb des Projektteams zu beachten.

Die Definition der Rolle der Wissenschaftler(innen) stellt eine Herausforderung für das transdisziplinäre Arbeiten dar. Jaeger-Erben et al. sprechen dabei von 
einer Hybridisierung der Rollen von Wissenschaftler(inne)n, die ,[...] oft neben der Erzeugung und Systematisierung von Wissen auch noch die Rolle von Moderatoren, Mediatoren, Supervisoren, Marketing- und Kommunikationsfachleuten, Pädagogen und noch viel mehr übernehmen. Diese Vielfalt an Rollen geht oftmals weiter [sic!] über das hinaus, was Wissenschaftler(innen) qua Expertise und Profession vertreten und verantworten können“ (2018, S. 120). Es bietet sich daher an, die Rollen der beteiligten Akteure vor Projektbeginn klar zu definieren und im Projektverlauf kontinuierlich zu überprüfen, ob diese noch eingehalten werden oder ob eine Anpassung der Rollenstrukturen vorgenommen werden muss. Zudem deutet diese Herausforderung auf die Relevanz der Schulung der Wissenschaftler(innen) bezüglich der verwendeten Methoden und Soft Skills hin.

Im Falle des Reallabors Schorndorf wurde ausgehend von der Projektkonzeption ein interdisziplinäres Wissenschaftsteam aus Ingenieur(inn)en, Psycholog(inn)en, Sozial- und Kommunikationswissenschaftler(inne)n sowie Stadtplaner(inne)n zusammengestellt, das alle wissenschaftlich-empirischen Arbeitsschritte (z. B. Datenanalyse, Interviews, Workshops) der TraSy-Methode durch seine Kompetenz und sein Methodenportfolio selbst durchführen konnte.

\subsection{Wie werden Einzelmethoden zur TraSy-Methode kombiniert?}

Die TraSy-Methode besteht aus einer Kombination von bereits existierenden Einzelmethoden, die für die Entwicklung eines komplexen Systems auf Basis heterogener Nutzeranforderungen gezielt zusammengestellt wurden. Bei der Zusammenstellung der Einzelmethoden zur TraSy-Methode wurde die Stimmigkeit der Methodenintegration und -rekombination beachtet, wie von Defila und Di Giulio (2018a, S. 51) gefordert. Die TraSy-Methode wurde gezielt für ein breites Anwendungsfeld entwickelt. Damit ist die Übertragbarkeit auf andere Themenbereiche gegeben (s. Abschnitt 1.2). Für die TraSy-Methode wurden qualitative und quantitative Methoden kombiniert. So wurde im Reallabor Schorndorf beispielsweise die quantitative Methode der Datenanalyse gewählt, um den Status quo in Bezug auf die soziodemografische Zusammensetzung der Bevölkerung Schorndorfs zu analysieren (s. Abschnitt 2.1). Der Großteil der Einzelmethoden in der TraSy-Methode beruht jedoch auf qualitativen Methoden empirischer Sozialforschung, die sich besonders dann eignen, wenn es darum geht, (noch) nicht (quantitativ) messbare Sachverhalte, Themen und Entwicklungen zu erfassen und zu explorieren (Grosvenor 2000; Røe 2000). Das Aufdecken subjektiver, handlungsleitender „Relevanzstrukturen hinter dem sichtbaren Verhalten“ (Scheiner 1998, S. 62f.) und das Verstehen psychologischer und sozialer Aspekte, die das Handeln der Menschen (mit-)bestimmen, kann nur mit Hilfe qualitativer Methoden erfolgen. 


\section{Der Weg zu neuen soziotechnischen Systemen mit Hilfe der TraSy-Methode}

Die TraSy-Methode wird so beschrieben, dass auch eine Anwendung auf andere Themenbereiche als den, der im Reallabor Schorndorf bearbeitet wurde, möglich ist. Dabei ist zu beachten, dass die Beschreibung der TraSy-Methode keine starre Methodenanleitung darstellt, sondern eine, die an den jeweiligen Kontext anzupassen ist. In Abhängigkeit des ,Umfangs' der Forschungsfrage muss deshalb beispielsweise die passende Anzahl und Länge der Workshops gewählt werden.

Abb. 1 zeigt den Weg von der Analyse des Handelns der potenziellen Nutzer(innen) des zu entwickelnden Systems (1) über die Identifikation von Nutzertypen und potenziellen Nutzungsszenarien im zukünftigen System (2) hin zur Generierung von Ideen und Konzepten (3) sowie zur Erprobung und Evaluierung (4) des neuen Systems. Unter Nutzungsszenarien sind dabei potenzielle Einsatzzwecke des zu entwickelnden Systems zu verstehen. Das im Folgenden beschriebene Vorgehen bezieht sich auf die Schritte 1-3. Die Erprobung des entwickelten Systems (4) ist nicht Gegenstand dieses Beitrags. An dieser Stelle soll jedoch betont

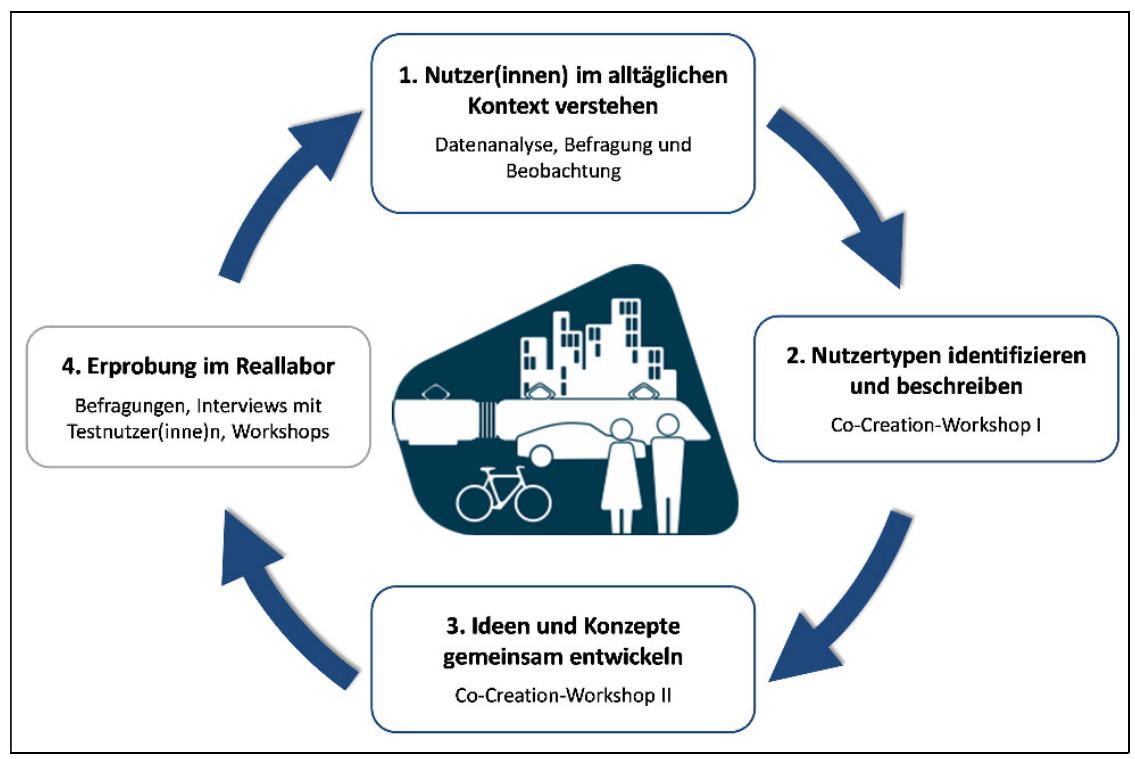

Abbildung 1: Schematische Darstellung der vier Phasen der TraSy-Methode; im Beitrag wird auf die Phasen 1-3 im Detail eingegangen. (C) Laura Gebhardt, Alexandra König. 
werden, dass ein mit der TraSy-Methode entwickeltes System unter Realbedingungen erprobt und validiert werden sollte. So testeten die Schorndorfer(innen) für neun Monate das im Reallabor Schorndorf mit der TraSy-Methode entwickelte Bussystem. In einem Pilotbetrieb vom März bis Dezember 2018 wurden die Nutzer(innen) intensiv wissenschaftlich begleitet, und das System wurde auf dieser Grundlage kontinuierlich evaluiert und angepasst (Brost et al. 2018; Gebhardt et al. 2019).

Das nachfolgend beschriebene methodische Vorgehen wird vor dem Hintergrund der gemachten Erfahrungen im Reallabor Schorndorf formuliert, es kann jedoch, wie in Abschnitt 1.2 gezeigt, auch in thematisch und räumlich andere Kontexte übertragen werden. Je nach Untersuchungsgegenstand bzw. Projektvorhaben gilt es, die Zeiträume, die für die einzelnen Phasen zur Verfügung stehen, anders zu definieren. Im Reallabor Schorndorf dauerte Phase 1 rund drei Monate, Phase 2 und Phase 3 dauerten von der Konzeption, über die Rekrutierung der Teilnehmer(innen) bis hin zur Durchführung und Auswertung jeweils rund fünf Monate, Phase 4 zehn Monate.

\subsection{Nutzer(innen) im alltäglichen Kontext verstehen}

Da die Methode stets in einem gesellschaftlichen Kontext angewendet wird, ist es wichtig, diesen Kontext annäherungsweise in seiner Komplexität zu verstehen. Ziel der ersten Phase der TraSy-Methode ist daher das Verstehen der Ausgangssituation, in die das zukünftige System eingebettet sein wird. Erfasst werden müssen zum Beispiel die raumstrukturellen Charakteristika (z. B. vorhandene Infrastruktur) und die soziodemografischen Besonderheiten des Untersuchungsgebiets. Diese Aufgabe wiederum wird von den Wissenschaftler(inne)n im Projektteam wahrgenommen. Dafür werden drei klassische Methoden empirischer Sozialforschung angewandt: 1) eine Daten- und Dokumentenanalyse, 2) eine teilnehmende Beobachtung und 3) Experteninterviews (s. Abb. 2).

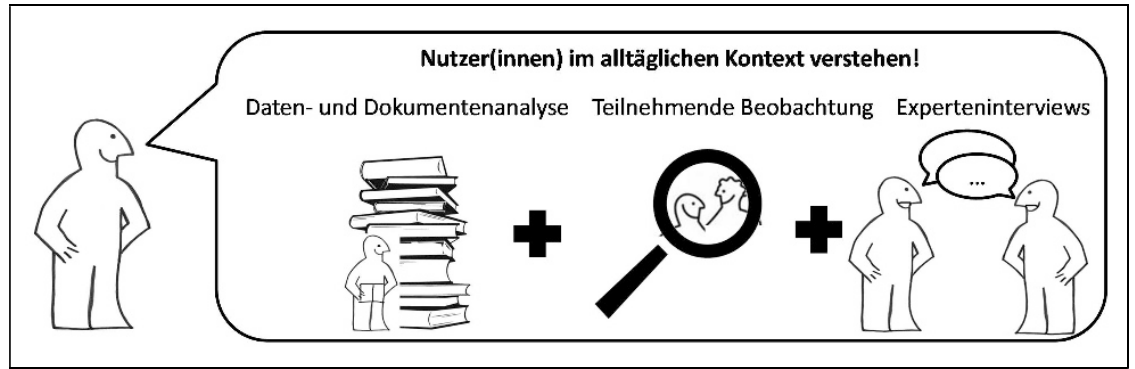

Abbildung 2: Grafische Darstellung der Arbeitsschritte in Phase 1 der TraSy-Methode. (C) Laura Gebhardt, Alexandra König. 
Alle Ergebnisse und Zwischenschritte werden systematisch dokumentiert. Dazu sieht die TraSy-Methode zwei zentrale Dokumente vor, ein Leitplankendokument sowie ein Spezifikationsheft. Im Leitplankendokument werden kontinuierlich während des gesamten Projektverlaufs Fragen gesammelt, die als relevant erachtet werden. Diese werden innerhalb des Projektteams regelmäßig diskutiert und bearbeitet. Das Leitplankendokument wird als Tabelle, mit Hilfe eines Tabellenkalkulationsprogramms o. Ä., erstellt (s. Abb. 3). Zu Beginn des Projekts ist das Leitplankendokument eine leere Tabelle. Alle zu diskutierenden Fragen werden einzeln in Tabellenzeilen notiert. In den dazugehörigen Spalten werden Ansprechpartner innerhalb oder außerhalb des Projektteams benannt. Zudem können neben der eigentlichen Beantwortung der Frage auch deren Bearbeitungsstatus sowie die Dringlichkeit der Frage festgehalten werden. Gemeinsam , abgesegnete Antworten werden hier festgehalten und dann in das Spezifikationsheft überführt.

Das Spezifikationsheft stellt eine Weiterentwicklung klassischer Lasten- und Pflichtenhefte dar, wie man sie in herkömmlichen Entwicklungs- und Produktionsprozessen kennt, d. h., es ist eine Zusammenführung aller für die Entwicklung des Systems relevanten Informationen. Es dient der Dokumentation der Ergebnisse aus allen Arbeiten, der Herleitung und Begründung der Parameterfestlegung (wie im Falle des Reallabors Schorndorf beispielsweise der Haltestellenabstände), dem Wissensaustausch zwischen den Projektbeteiligten sowie der Vereinheitlichung des Kenntnisstandes durch fortgesetztes Einarbeiten der Ergebnisse von Absprachen und Berichten.

Am Anfang des Dokuments werden die Projektziele aufgelistet und der Zeitplan eingetragen. Weiterhin werden die (z. B. von Teilprojekten) erwarteten Teilergebnisse benannt. Zu Beginn des Projekts enthält das Spezifikationsheft ausschließlich diese Inhalte. Im weiteren Verlauf wird das Herzstück des Dokuments ergänzt: die Beschreibung der Spezifikationen des zu entwickelnden Systems. Die Spezifikationen sind detaillierte Beschreibungen der Systemeigenschaften (im Reallabor Schorndorf gehörte dazu z. B. der maximale Haltestellenabstand zwischen den einzelnen Haltestellen). Die Spezifikationen werden, so detailliert wie möglich, in thematisch abgegrenzten Kapiteln festgehalten, die in ihrer Gesamtheit einen Überblick über das System als Ganzes bieten. Das Spezifikationsheft wird nach der Durchführung jeder der Einzelmethoden anhand der neuen Erkenntnisse und Ergebnisse durch das Projektteam aktualisiert. Ergebnisse werden so fortlaufend festgehalten und innerhalb des Projektteams in Treffen und regelmäßigen Telefonkonferenzen diskutiert, die bestenfalls zweiwöchentlich stattfinden sollten und deren Länge von den jeweils zu besprechenden Themen abhängt. In diesen Besprechungen wird entschieden, welche Ergebnisse, d. h. welche Spezifikationen in das Spezifikationsheft aufgenommen werden sollen. Das Spezifikationsheft dient zum Beispiel auch zur Beauftragung von Unteraufträgen (wie z. B. Programmiertätigkeiten). 


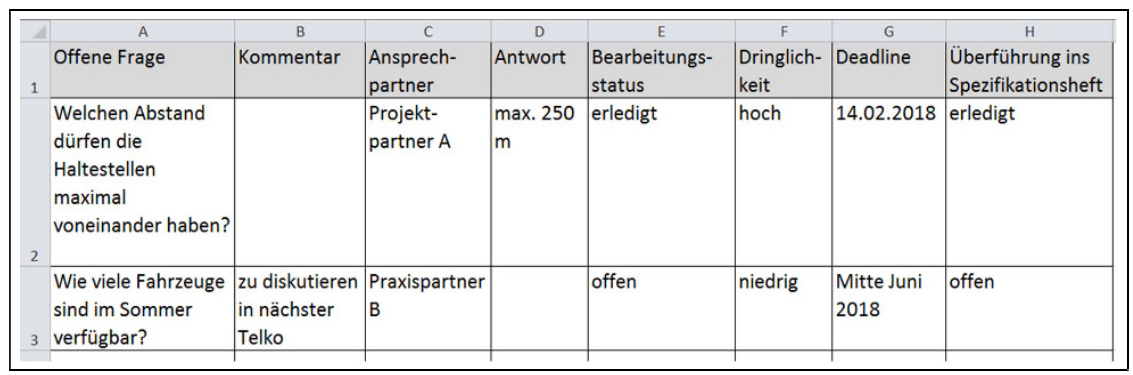

Abbildung 3: Beispiel eines Leitplankendokuments. (C) Laura Gebhardt, Alexandra König.

\subsubsection{Daten- und Dokumentenanalyse}

Ausgehend von der Annahme der handlungsorientierten Forschung, dass Menschen ihr Handeln unter den Bedingungen ihrer Lebenswelt produzieren und reproduzieren, werden das alltägliche Handeln der Nutzer(innen) und dessen Handlungsrahmen als Ausgangspunkt für die Entwicklung des neuen Systems in den Blick genommen. Konkret bedeutet dies, dass soziodemografische und räumliche Daten recherchiert, ausgewertet und interpretiert werden. Die Dokumentenanalyse sollte von der Frage geleitet sein, welche Daten wichtig sind, um den Status quo hinsichtlich des Projektziels zu verstehen. Dabei sollte aber stets dem Prinzip gefolgt werden, dass es umso besser ist, je mehr Informationen über das zu untersuchende Gebiet, seine Charakteristika und die darin handelnden Menschen gesammelt und ausgewertet werden. Für die Daten- und Dokumentenanalyse bieten die jeweiligen nationalen statistischen Ämter oder kommunalen Datenbestände, im Falle Deutschlands das Statistische Bundesamt oder das Bundesinstitut für Bau-, Stadt- und Raumforschung, eine solide Basis solcher Daten.

Zunächst gilt es, die relevanten und zu betrachtenden Parameter zu identifizieren. Da die TraSy-Methode soziotechnische Systeme entwickelt, sind soziodemografische Daten stets zu recherchieren. Alle weiteren relevanten Parameter hängen von dem zu entwickelnden System ab. Bei einem Vorhaben im Bereich Mobilität macht es beispielsweise Sinn, sich das aktuelle Mobilitätsverhalten im Untersuchungsraum anzusehen. Bei der Betrachtung ist immer auch von Bedeutung, die recherchierten Zahlen in Vergleich zu anderen Räumen (z. B. der gesamtdeutsche Durchschnitt) zu setzen, um ein besseres Verständnis für die spezifischen Charakteristika des Untersuchungsgebiets (z. B. der überdurchschnittliche Altersdurchschnitt der Bevölkerung) zu bekommen.

Zentrale Fragen im Reallabor Schorndorf waren beispielsweise: Wer wohnt hier? Welche Verkehrsmittel werden derzeit bevorzugt genutzt? Wie gut ist der öffent- 


\section{Altersstruktur:}

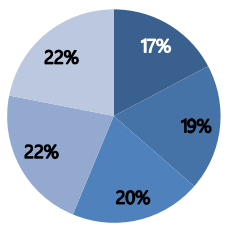

unter 18

$\square$ - 18 bis unter 35

35 bis unter 50

50 bis unter 65

65 und älter

$\mathrm{N}=39.520$

Das Durchschnittsalter in Schorndorf beträgt 51,5 Jahre.

Der Bundesdurchschnitt liegt bei 44,3 Jahren.

\section{Pkw-Besitz:}

Deutschland (gesamt)

Schorndorf

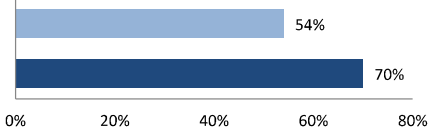

\section{Modal Split:}

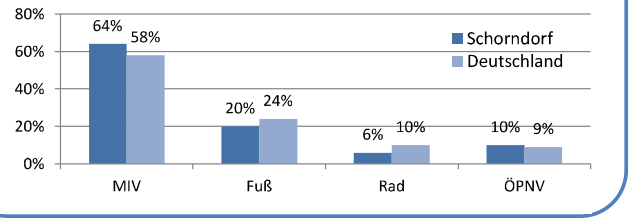

\section{Haushaltsmerkmale:}

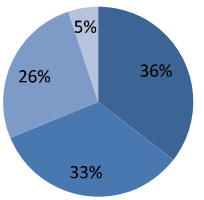

$$
\begin{aligned}
& \text { Einpersonenhaushalte } \\
& \text { Zweipersonenhaushalte } \\
& \text { Drei- und Vierpersonenhaushalte } \\
& \text { Haushalte mit } 5 \text { und mehr Personen } \\
& N=17.891
\end{aligned}
$$

Im Durchschnitt leben 2,2 Personen in einem Haushalt.

Der Bundesdurchschnitt liegt bei 2 Personen.

Abbildung 4: Relevante Kriterien für die Daten- und Dokumentenanalyse sowie beispielhafte Ergebnisse für das im Reallabor Schorndorf untersuchte Thema. Von oben: Altersverteilung der Bevölkerung in Schorndorf nach Altersgruppen; Pkw-Besitz in Schorndorf im bundesdeutschen Vergleich; Verkehrsmittelnutzung in Schorndorf im bundesdeutschen Vergleich; Haushaltsstruktur in Schorndorf. Quellen: Verband Region Stuttgart 2011, Mobilität in Deutschland 2008. C) Laura Gebhardt, Alexandra König. 
liche Verkehr? Die Abb. 4 illustriert beispielhaft für das Vorhaben in Schorndorf die relevanten Parameter sowie Ergebnisse der Dokumentenanalyse. Für die Analyse der Ausgangssituation in Schorndorf wichtige Parameter waren etwa die Altersstruktur und die aktuelle Verkehrsmittelnutzung.

\subsubsection{Teilnehmende Beobachtung}

Um ein über die Analyse von statistischen Daten hinausgehendes Verständnis für die Rahmenbedingungen vor Ort zu erlangen, finden teilnehmende Beobachtungen statt. Das Kennzeichnende dieser Methode ist die persönliche Teilnahme der Forschenden an Interaktionen im Untersuchungsraum. Dabei ist die Annahme leitend, dass durch die dabei mögliche unmittelbare Erfahrung der Situation Aspekte des Handelns und Denkens beobachtbar werden, die in Gesprächen und Dokumenten über diese Interaktionen bzw. Situationen nicht zugänglich wären (Girtler 2002; Lüders 2003).

Damit gute Ergebnisse erzeugt werden, müssen die Ziele der teilnehmenden Beobachtungen im Vorfeld klar definiert werden, so dass die Beobachter(innen) wissen, auf was sie achten müssen. Zudem muss eine Dokumentationsform bestimmt werden, mit deren Hilfe die gemachten Beobachtungen festgehalten werden. Hier bietet sich ein vorstrukturiertes Beobachtungsprotokoll an. Dieses sollte geschlossene Fragen enthalten (im Reallabor Schorndorf z. B. „Wie viele Fahrgäste waren im Bus anwesend?"), aber auch die Möglichkeit für Notizen über weiterführende Beobachtungen in Freitextfeldern lassen. Da das Vorgehen einen explorativen Charakter besitzt, muss flexibel auf Veränderungen eingegangen werden können. Das bedeutet, dass während der Beobachtung weitere Fragen in das Beobachtungsprotokoll aufgenommen werden sollten, wenn sich Hinweise auf deren Relevanz ergeben. Die teilnehmende Beobachtung sollte verteilt auf mehrere Tage stattfinden, um ein möglichst genaues Bild der Situation zu erhalten. Zudem sollten sich mindestens zwei Beobachter(innen) ein Bild von der Lage machen und ihre Ergebnisse unabhängig voneinander dokumentieren und auswerten. Die Auswertung sollte zwar von den vorher definierten Forschungsfragen geleitet werden, jedoch sollte die teilnehmende Beobachtung auch genutzt werden, um neue Hinweise zu erhalten, die in den anschließenden Experteninterviews diskutiert werden können.

Im Reallabor Schorndorf wurden Vor-Ort-Begehungen von Haltestellen und Probefahrten mit den bestehenden innerstädtischen Buslinien vorgenommen. Das Ziel der teilnehmenden Beobachtungen war die Erfassung von Informationen zu folgenden Punkten: Pünktlichkeit, Fahrgastaufkommen und -struktur, die Situation beim Ein- und Ausstieg, Möglichkeiten zum Festhalten, Gangbreite und Situation beim Durchgehen nach hinten, Platzverhältnisse (für Kinderwagen, Rollstühle etc.), Fahrkomfort, Ticketkauf sowie allgemeine Stimmung und Interaktionen zwischen den Fahrgästen. 


\subsubsection{Experteninterviews}

Um die in den vorausgehenden Schritten gesammelten Daten und Einsichten zu untermauern, werden Interviews mit Personen vor Ort geführt. Als Expert(inn)en werden Personen mit Vorwissen zum jeweiligen Themenfeld, in das das Projektvorhaben eingebettet ist, ausgewählt. Dabei gilt es, Expertengespräche mit Personen aus unterschiedlichen Bereichen zu führen, um eine möglichst große Bandbreite an Einschätzungen einzufangen. Die Gespräche haben zum Ziel, explorative Erkenntnisse über die Gegebenheiten im Untersuchungsraum zu erhalten, um den Rahmen, in dem das System angewendet werden soll, möglichst gut zu kennen. Zudem wird mit den Gesprächen die Bildung eines lokalen Netzwerkes an Expert(inn)en angestrebt, die auch für weitere Fragen konsultiert werden und die Einführung des neuen Systems unterstützen können. Das Hauptkriterium für die Auswahl der Expert(inn)en ist deren Zugang zu spezifischem Wissen, das für die Systementwicklung von Bedeutung sein könnte. Die Befragung der Expert(innen)en dauert ungefähr eine Stunde und basiert auf einem teilstrukturierten Leitfaden, der sowohl für alle identische Fragen enthält als auch solche, die zugeschnitten sind auf den Kompetenzbereich des/der jeweiligen Experten(in) (für ein Beispiel aus dem Reallabor Schorndorf, s. Anlage a im Anhang dieses Beitrags). Für diese Arbeiten ist es wichtig, Mitarbeiter(innen) mit Erfahrungen in der Anwendung von Methoden der empirischen Sozialforschung im Projekt zu haben.

Die Auswertung der Expertenbefragung erfolgt mit Blick auf die Identifizierung potenzieller Nutzertypen sowie möglicher Nutzungsszenarien. Diese fließen als Input in Phase 2 der TraSy-Methode ein. Die Erkenntnisse aus Phase 1 werden in die beiden zentralen Dokumente zur Wissensbündelung innerhalb des Gesamtprojekts - das Leitplankendokument und das Spezifikationsheft - überführt.

Im Reallabor Schorndorf wurden die Experteninterviews mit Vertreter(inne)n des Verkehrsclubs Deutschland (VCD), des Allgemeinen Deutschen Fahrrad-Clubs (ADFC), eines lokalen Gewerbevereins (Centro), von Stadtmobil, des Seniorenforums, eines lokalen Taxiunternehmens, eines lokalen Busunternehmens und des Gemeinderats durchgeführt. Mit Hilfe der Expert(inn)en wurden Erkenntnisse gesammelt über aktuelle Schwachstellen und Nutzungshürden im Zusammenhang mit dem öffentlichen Nahverkehr sowie Erkenntnisse über mögliche Zielgruppen des neuen Buskonzepts (Senior(inn)en, mobilitätseingeschränkte Personen, regelmäßige Autonutzer(innen), regelmäßige Busnutzer(innen)). 


\subsection{Nutzertypen identifizieren und beschreiben- Co-Creation-Workshop I}

In dieser Phase geht es darum, die in Phase 1 benannten möglichen Nutzertypen und Nutzungsszenarien vertiefend zu prüfen und zu charakterisieren. Dies erfolgt nun gemeinsam mit Praxisakteuren.

Von entscheidender Bedeutung für den Erfolg von Methoden, die auf die Einbeziehung von Praxisakteuren (insbes. aus der Zivilgesellschaft) ausgerichtet sind, erweisen sich Dialog-Methoden, die einen „kommunikativen Raum“ (Bergold und Thomas 2010, S. 337) für die Wissensgenerierung schaffen (Defila und Di Giulio 2018a, S. 45). Daher sieht die TraSy-Methode im Anschluss an die beschriebene Analyse der Ausgangssituation einen Co-Creation-Workshop vor. CoCreation beschreibt dabei einen kreativen und wechselseitigen Lernprozess, in den sowohl wissenschaftliche als auch außerwissenschaftliche Akteure involviert sind, mit dem Ziel, gemeinsame Ergebnisse zu generieren.

Der im Folgenden beschriebene Workshop I dient dazu, die unterschiedlichen Nutzertypen und deren Anforderungen an das zu entwickelnde System zu verstehen und potenzielle Nutzungsszenarien zu entwickeln. Zentrale Herausforderungen, deren man sich vorab bewusst sein sollte, sind dabei die Heterogenität der Akteure und die unterschiedlichen Anforderungen verschiedener Akteursgruppen, die sich zum Teil auch widersprüchlich gegenüberstehen. Dieser Herausforderung kann durch die Arbeit mit Nutzergruppen begegnet werden. Eine Nutzergruppensegmentierung stellt die Möglichkeit dar, die Komplexität heterogener Populationen durch die Identifikation von homogenen Subgruppen zu reduzieren (für eine Übersicht über Typisierungsansätze s. Hunecke 2015). Dadurch kann ein tiefgehendes Verständnis der Bedürfnisse einzelner Gruppen erzeugt und bei der Entwicklung des Systems berücksichtigt werden.

Dieser erste Workshop dient also dazu, die unterschiedlichen Nutzergruppen und deren Anforderungen an das zu entwickelnde System zu verstehen und potenzielle Nutzungsszenarien gemeinsam zu erarbeiten. Die Vorbereitung, Durchführung und Auswertung des Workshops sind im Folgenden in Bezug auf das Themenfeld Mobilität beschrieben, können jedoch analog auch in anderen Themenfeldern angewandt werden.

\subsubsection{Workshopziele}

Wie bereits dargelegt, hat der Co-Creation-Workshop I zum Ziel, die Nutzer(innen) bei der Ausgestaltung des Systems in einer frühen Entwicklungsphase zu involvieren, d. h., die nutzertypenspezifischen Anforderungen zu identifizieren. Auf Basis dieser Anforderungen können anschließend konkrete Entwürfe des Systems entstehen, die beschreiben, welche Eigenschaften es besitzen sollte, d. h., was es 
leisten muss, um den Anforderungen der unterschiedlichen Nutzertypen zu entsprechen.

Der Workshop hat folgende Ziele:

1) Spezifizierung und Validierung der Nutzertypen

2) Skizzierung einer prototypischen Nutzung des neuen Systems durch unterschiedliche Nutzertypen

3) Entwicklung und Beschreibung von Funktionsweisen des neuen Systems

Für die Entwicklung des Bussystems im Reallabor Schorndorf bedeutete dies: 1) detaillierte Beschreibung potenzieller Nutzergruppen des neuen Bussystems; 2) Skizzierung einer fiktiven Nutzung des Busses (Bedarfsermittlung, Reiseplanung und Reisedurchführung, s. Abb. 7); 3) Festlegung von Abholpunkten, Betriebszeiten und weiteren Funktionalitäten des Systems.

\subsubsection{Vorphase: Bisher identifiziertes Wissen nutzbar machen}

Die Ergebnisse der vorherigen Phase Nutzer(in) im alltäglichen Kontext verstehen (s. Abschnitt 2.1) werden in Form der im Leitplankendokument erfassten Antworten als Input für den Workshop verwendet (s. Abb. 5). Diese Antworten stellen wichtige Leitplanken dar, die der Arbeit im Workshop einen Rahmen geben sollen. Offene Fragen aus dem Leitplankendokument werden im Workshop adressiert, um Antworten zu finden. Die Leitplanken sind beispielsweise bestimmte Themen oder Fragestellungen, die durch das Projektvorhaben nicht beeinflusst werden können. Diese Restriktionen müssen zusammengestellt werden, um sie den Workshopteilnehmer(inne)n kommunizieren zu können. Im Reallabor Schorndorf war dies zum Beispiel die Frage nach dem Betriebsgebiet innerhalb der Stadt. Im Rahmen der Untersuchungen in Phase 1 hatte sich klar herausge-

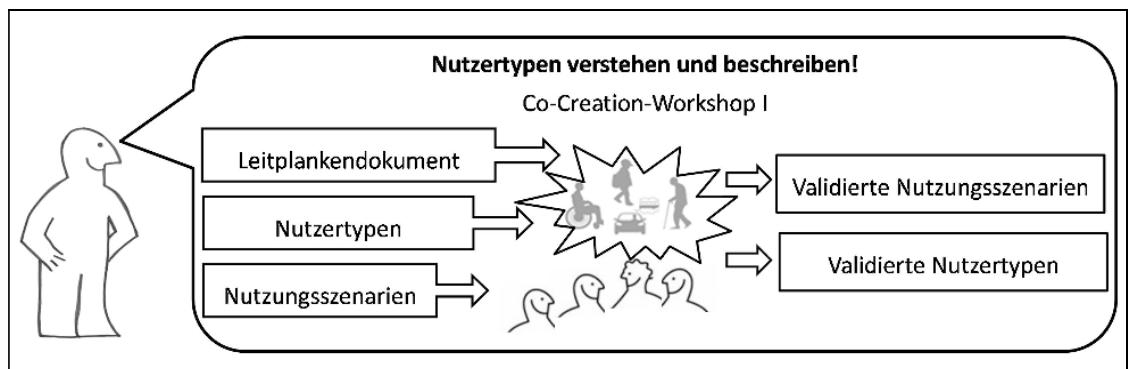

Abbildung 5: Co-Creation-Workshop I zur Validierung von Nutzertypen und Nutzungsszenarien. (C) Laura Gebhardt, Alexandra König. 
stellt, dass eine Einbindung aller Teilorte Schorndorfs aus technischen und organisatorischen Gründen nicht möglich sein würde. Diese Restriktion wurde in Form der Festlegung des in Frage kommenden Einsatzgebiets für das neue Bussystem von Anfang an kommuniziert.

Weiterhin ist das Wissen über Nutzertypen und deren Handeln, das in der vorherigen Phase (s. Abschnitt 2.1) generiert wurde, so aufzubereiten, dass es den Workshopteilnehmer(inne)n verständlich präsentiert werden kann. Dies geschieht in Form von grafisch aufbereiteten Profilen der identifizierten Nutzertypen (s. Abb. 6). Gleiches gilt für die angedachten Nutzungsszenarien (s. Abb. 7). Beide werden im Verlauf des Workshops weiter ausgestaltet.

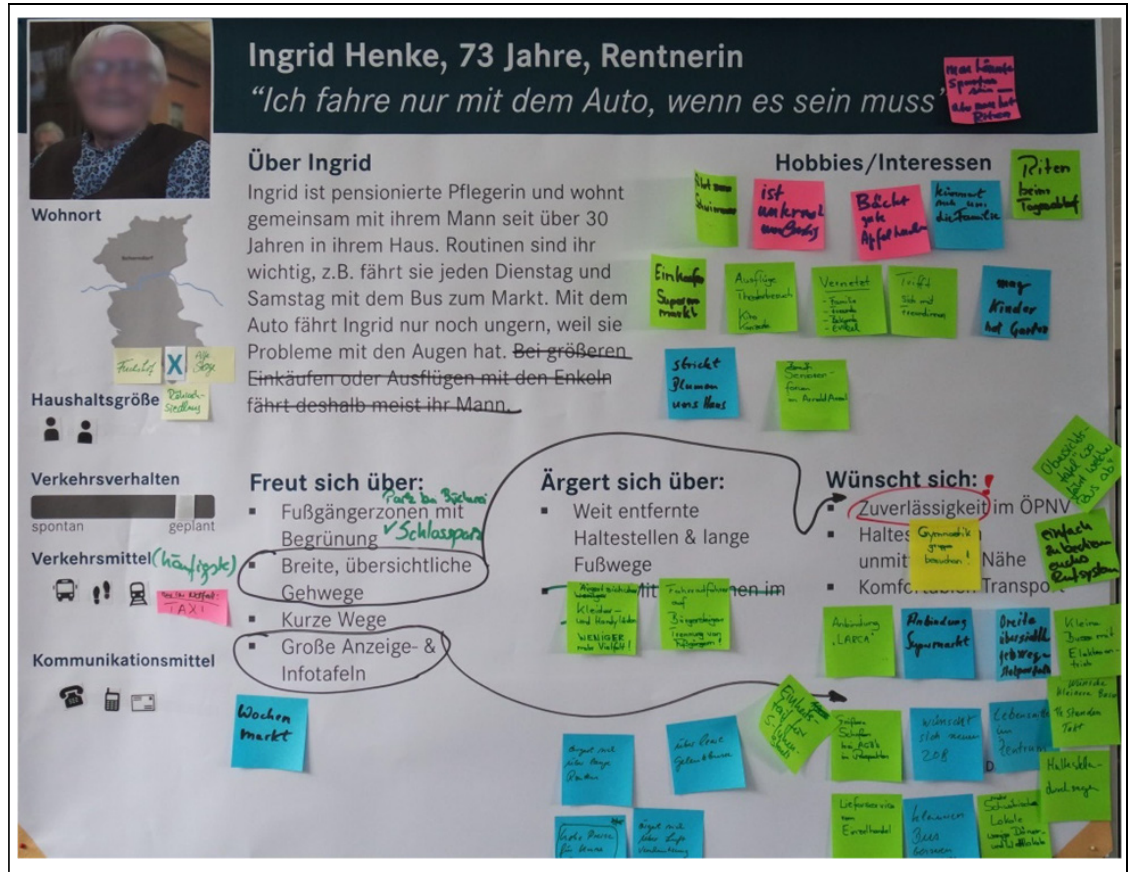

Abbildung 6: Die gedruckten Informationen basieren auf dem in Phase 1 gewonnenen Wissen über Nutzertypen. Sie dienen als Grundlage zur strukturierten Diskussion der Nutzertypen. Durch Beschriftungen, Klebezettel, Symbole (z. B. Darstellung der Verkehrsmittel) wird das Nutzerprofil gemeinsam weiterentwickelt, hier am Beispiel der Seniorin Frau Henke. (C) Salome Keller. 


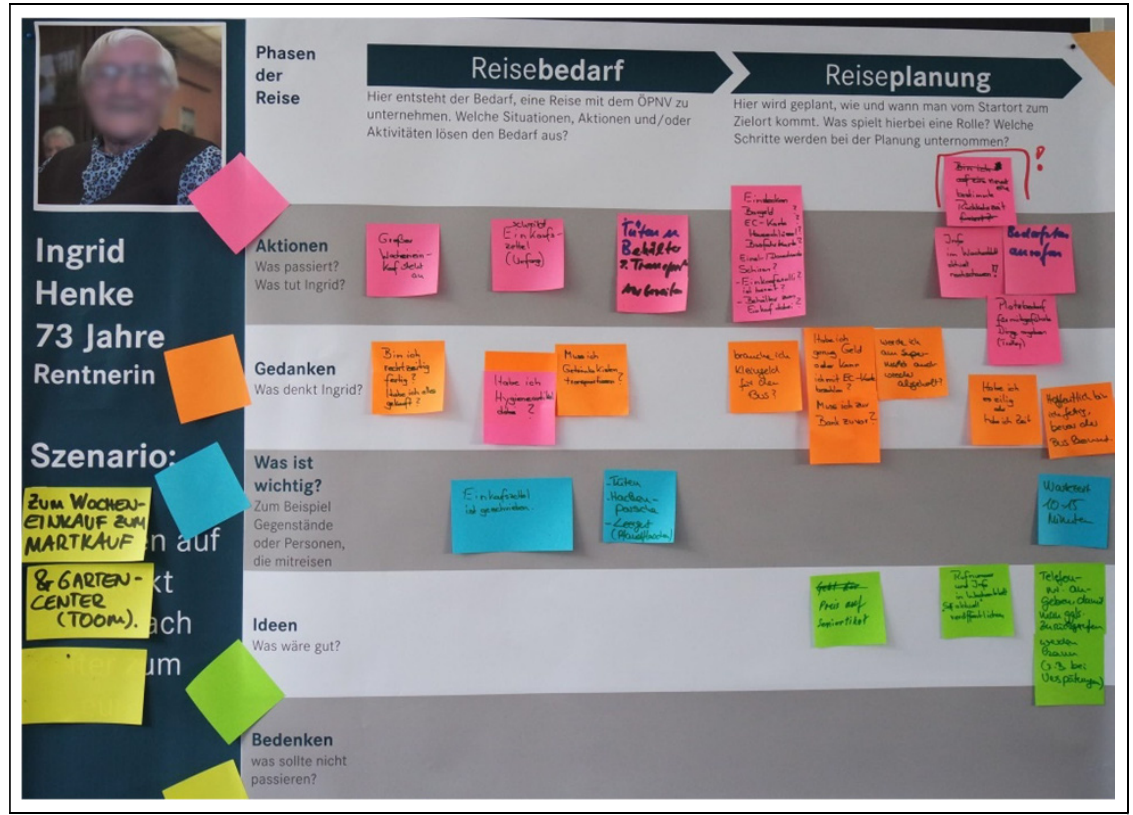

Abbildung 7: Die gedruckten Informationen basieren auf dem in Phase 1 gewonnenen Wissen über Nutzungsszenarien. Sie dienen als Vorlage zur Diskussion eines Nutzungsszenarios. Bezogen auf einen Nutzertyp - hier die Seniorin Frau Henke - wird der Reiseablauf schrittweise durchlebt und davon ausgehend prototypisch beschrieben. (C) Salome Keller.

\subsubsection{Inhaltliche und methodische Vorbereitung}

Mit Blick auf die methodische Vorbereitung sollte ein Regiebuch zur Planung des Workshopablaufs erstellt werden. Dieses Regiebuch kann eine einfache Tabelle mit Zeitangaben sowie Arbeitsaufträgen für und Leitfragen an die Teilnehmer(innen) sein, das die Moderator(inn)en unterstützt und gewährleistet, dass die Arbeit in Kleingruppen analog zueinander abläuft.

Wie bereits unter Vorphase: Bisher identifiziertes Wissen nutzbar machen (s. Abschnitt 2.2.2) genannt, gilt es vor allem, das Inputmaterial für den Workshop zielgruppenspezifisch aufzubereiten. Hier geht es in erster Linie um eine Komplexitätsreduktion. Es gilt, die relevanten Informationen und $\mathrm{zu}$ diskutierenden Themen und Fragestellungen verständlich aufzubereiten. Dies betrifft sowohl das Wissen um Nutzertypen und Nutzungsszenarien als auch die für das Projekt - und damit die Diskussion im Workshop - geltenden Restriktionen. Im Workshop des Reallabors Schorndorf erleichterte zum Beispiel ein großer Stadtplan die Diskussion um das Einsatzgebiet des Busses. 
Zur Strukturierung des Workshops sind zudem Visualisierungen zu empfehlen, die vorab erstellt werden sollten. Neben der Agenda (s. Abb. 8) können beispielsweise auch die Anleitung zur Vorstellungsrunde (Mein Name ist...; Ich wohne in...; Mein eindrucksvollstes Erlebnis im Zusammenhang mit...) oder Arbeitsaufträge entsprechend gestaltet werden.

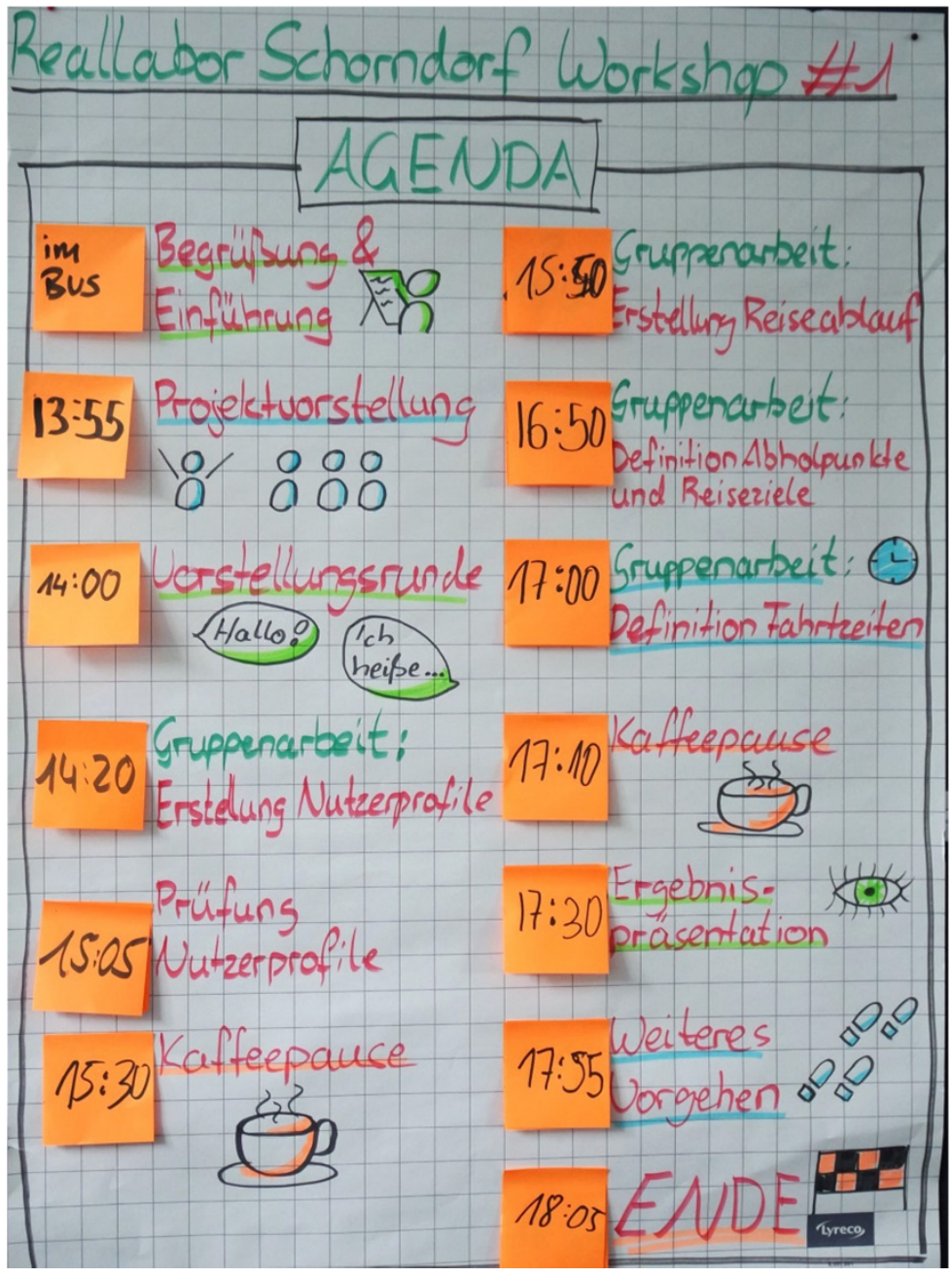

Abbildung 8: Illustrierter Ablauf des Workshops. Post-its bieten die Möglichkeit, auf Veränderungen, die nötig werden, agil zu reagieren und ggf. die Zeiten anzupassen. (C) Salome Keller. 
Für die Auswahl und Vorbereitung des Tagungsorts schließlich sind die folgenden Punkte zu bedenken: Es empfiehlt sich, zentral gelegene Räumlichkeiten zu nutzen, die für die Teilnehmer(innen) gut zu erreichen sind und genügend Platz sowohl für die Arbeit im Plenum als auch für die Arbeit in Kleingruppen bieten. Idealerweise lässt sich zusätzlich ein Eingangsbereich (Foyer) so gestalten, dass hier informelle Gespräche nach dem offiziellen Workshop stattfinden können. Das Anbringen eines beschreibbaren Posters mit der Überschrift „Themenspeicher" erweist sich als sinnvoll, um Anregungen und Fragen zu sammeln, für die im Rahmen des Workshops kein oder zu wenig Raum ist.

\subsubsection{Ansprache und Gewinnung der Teilnehmer(innen)}

Für die Motivation und Gewinnung der Teilnehmer(innen) ist es sinnvoll, Praxisakteure bei lokalen Einrichtungen anzusprechen. Zusätzlich kann die Rekrutierung durch Anzeigen in verschiedenen Medien (Wochenzeitung, Projekthomepage, Facebook, Twitter) oder durch eine Zufallsstichprobenziehung (nach Alter und Geschlecht) durch das Einwohnermeldeamt (soweit es um die Bevölkerung geht) und das Anschreiben der gezogenen Personen gelingen. Am Workshop sollten zwischen 15 und 20 Praxisakteure teilnehmen, deren Teilnahme vor dem Workshop bestätigt sein sollte. Diese Gruppengröße erlaubt es, sowohl intensiv in Kleingruppen (à 4-5 Personen) als auch im Plenum zu diskutieren.

Im Rahmen der Ansprache und Gewinnung der Teilnehmer(innen) wird kommuniziert, dass es Bild- und Tonaufnahmen geben wird, die lediglich für interne Zwecke verwendet werden. Innerhalb des Workshops wird dies den Teilnehmer(inne)n noch einmal schriftlich vorgelegt, mit der Bitte, sich durch ihre Unterschrift damit einverstanden zu erklären.

Ausreichende Informationen bezüglich des Projektvorhabens und der Projektziele sind wichtig für die Partizipationsbereitschaft der Praxisakteure. Dafür bietet es sich an, mit einer Werbeagentur eine umfangreiche Informations- und Werbekampagne zu gestalten. Hierfür sollte eine enge Zusammenarbeit zwischen Projektteam und Agentur stattfinden, um zu erörtern, welche Gruppen über welche Kanäle erreicht werden können. Ältere Menschen können beispielsweise eher über Zeitungen und Informationsveranstaltungen erreicht werden, wohingegen Kinder und Jugendliche eher über Vereine, Jugendzentren, Schulen etc. angesprochen werden können. Vereine und Verbände sind darüber hinaus wichtige Multiplikatoren. Wichtig ist, dass keine für das Thema relevante Gruppe ausgeschlossen wird und dass darauf geachtet wird, Zugangsbarrieren (z. B. sprachlicher Natur) zu vermeiden. 
Im Reallabor Schorndorf wurden alle hier genannten Kanäle zur Rekrutierung der Teilnehmer(innen) genutzt. Der Teilnehmerkreis setzte sich schließlich zur Hälfte aus Personen zusammen, die basierend auf einer Zufallsstichprobenziehung durch das Einwohnermeldeamt der Stadt Schorndorf angeschrieben wurden, zur anderen Hälfte aus Personen, die sich aufgrund der örtlichen Informationen angesprochen fühlten.

\subsubsection{Gruppeneinteilung und Moderation}

Das Workshopkonzept sieht vor, dass es innerhalb des Workshops Phasen in der Gesamtgruppe und Phasen in Kleingruppen gibt. Die Kleingruppen setzen sich zum einen aus Vertreter(inne)n der Nutzertypen zusammen (pro Nutzertyp eine Gruppe). Zusätzlich sollte es in jeder Kleingruppe eine(n) Wissenschaftler(in) sowie eine(n) Moderator(in) geben. Im Idealfall hat das Projektteam Mitglieder, die über Moderationskompetenzen verfügen. Sollte dies nicht der Fall sein, sollten externe Moderator(inn)en engagiert werden.

\subsubsection{Durchführung des Workshops}

Der Ablaufplan (s. Tab. 1) zeigt, wie sich innerhalb des rund vierstündigen Workshops Phasen in der Gesamtgruppe und Gruppenarbeitsphasen ergänzen. Tab. 1 zeigt, welche Informationen, Leitfragen bzw. Arbeitsaufträge in den unterschiedlichen Phasen relevant sind. Die Formulierung der Fragen bzw. Arbeitsaufträge hängt selbstverständlich vom jeweiligen thematischen Kontext des zu entwickelnden Systems ab.

Neben einer Feedback-Runde im Plenum am Ende des Workshops bietet sich eine (anonymisierte) Abfrage per Fragebogen im Anschluss an den Workshop an, um Hinweise zur individuell-subjektiven Bewertung der entwickelten Ideen zu erhalten.

Im Reallabor Schorndorf wurden die Teilnehmer(innen) zum Beispiel gefragt, inwieweit sie sich mit dem entworfenen Nutzertyp und dem zugehörigen Nutzungsszenario identifizieren können bzw. welche Aspekte sie gerne noch in die Diskussion und den Entwicklungsprozess eingebracht hätten (zur Veranschaulichung s. Fragebogen in Anlage b im Anhang dieses Beitrags). So ließ sich feststellen, dass der Großteil der Teilnehmer(innen) sich sowohl mit den Nutzerprofilen als auch den potenziellen Nutzungsszenarien stark identifizieren konnte. 
Tabelle 1: Ablaufplan des Co-Creation-Workshops I inklusive Leitfragen und Arbeitsanweisungen.

\begin{tabular}{|c|c|c|c|}
\hline Phase & Was ist zu tun? & $\begin{array}{l}\text { Fragen } \& \\
\text { Arbeitsanweisungen }\end{array}$ & $\begin{array}{l}\text { Zeitfenster } \\
\text { in Minuten }\end{array}$ \\
\hline $\begin{array}{l}\text { Begrüßung } \\
\text { und Projekt- } \\
\text { vorstellung }\end{array}$ & $\begin{array}{l}\text { Begrüßung, Nen- } \\
\text { nung der Veranstal- } \\
\text { tungsziele \& Agen- } \\
\text { da, Kurzpräsenta- } \\
\text { tion des Projekts }\end{array}$ & $\begin{array}{l}\text { Alltagssprache wählen, um } \\
\text { das Projektvorhaben zu } \\
\text { skizzieren (im Stil von „was } \\
\text { bisher geschah“, ohne zu } \\
\text { viele Projektdetails aufzu- } \\
\text { führen) } \\
\text { Hinweis auf Themen- } \\
\text { speicher }\end{array}$ & 10 \\
\hline $\begin{array}{l}\text { Vorstellungs- } \\
\text { runde }\end{array}$ & $\begin{array}{l}\text { Das Eis brechen: } \\
\text { Einladen zur Vor- } \\
\text { stellungsrunde; Mo- } \\
\text { derator(in) startet } \\
\text { mit einem positiven } \\
\text { Beispiel }\end{array}$ & $\begin{array}{l}\text { Mein Name ist ... / Ich woh- } \\
\text { ne in ... / Mein ein- } \\
\text { drucksvollstes Erlebnis im } \\
\text { Zusammenhang mit ... (pas- } \\
\text { send zum Themenfeld wäh- } \\
\text { len) }\end{array}$ & $\begin{array}{l}20, \text { ca. } \\
1 \text { Min. pro } \\
\text { Teilneh- } \\
\text { mer(in) }\end{array}$ \\
\hline $\begin{array}{l}\text { Gruppenarbeit: } \\
\text { Prüfung und } \\
\text { Ausdifferen- } \\
\text { zierung Nutzer- } \\
\text { profile }\end{array}$ & $\begin{array}{l}\text { Pro Gruppe das } \\
\text { idealtypisch skiz- } \\
\text { zierte Nutzerprofil } \\
\text { diskutieren, ändern, } \\
\text { ergänzen, spezifi- } \\
\text { zieren }\end{array}$ & $\begin{array}{l}\text { Entspricht die Beschreibung } \\
\text { des Nutzertyps Ihnen? Wel- } \\
\text { che Aspekte fehlen oder } \\
\text { sind nicht stimmig? Über } \\
\text { was freut sich Frau/Herr X, } \\
\text { was ärgert sie/ihn, was } \\
\text { wünscht sie/er sich? usw. } \\
\text { (für ein Beispiel des Ergeb- } \\
\text { nisses s. Abb. 6) }\end{array}$ & 45 \\
\hline Pause & $\begin{array}{l}\text { Imbiss/Getränke } \\
\text { bereitstellen, an } \\
\text { Themenspeicher } \\
\text { erinnern }\end{array}$ & $\begin{array}{l}\text { Informelle Gespräche mit } \\
\text { den Teilnehmer(inne)n } \\
\text { suchen }\end{array}$ & 20 \\
\hline $\begin{array}{l}\text { Gruppenarbeit: } \\
\text { Prüfung und } \\
\text { Ausdifferenzie- } \\
\text { rung Nutzungs- } \\
\text { szenarien }\end{array}$ & $\begin{array}{l}\text { Pro Gruppe das für } \\
\text { den entsprechen- } \\
\text { den Nutzertyp skiz- } \\
\text { zierte Nutzungs- } \\
\text { szenario diskutie- } \\
\text { ren, ändern, ergän- } \\
\text { zen, spezifizieren }\end{array}$ & $\begin{array}{l}\text { Zu welchem Zweck könnte } \\
\text { der Nutzertyp das System } \\
\text { nutzen? Welche Gedanken } \\
\text { würden einer Person bei der } \\
\text { Nutzung durch den Kopf } \\
\text { gehen? Was ist ihr dabei } \\
\text { wichtig? Was bereitet Sor- } \\
\text { ge? usw. (für ein Beispiel } \\
\text { des Ergebnisses s. Abb. 7) }\end{array}$ & 50 \\
\hline
\end{tabular}




\begin{tabular}{|c|c|c|c|}
\hline Phase & Was ist zu tun? & $\begin{array}{l}\text { Fragen \& } \\
\text { Arbeitsanweisungen }\end{array}$ & $\begin{array}{l}\text { Zeitfenster } \\
\text { in Minuten }\end{array}$ \\
\hline $\begin{array}{l}\text { Gruppenarbeit: } \\
\text { Benennung von } \\
\text { konkreten } \\
\text { Funktionen des } \\
\text { neuen Systems }\end{array}$ & $\begin{array}{l}\text { Pro Gruppe die für } \\
\text { den Nutzertyp rele- } \\
\text { vanten Eigenschaf- } \\
\text { ten des Systems } \\
\text { definieren }\end{array}$ & $\begin{array}{l}\text { Wie müsste das System ge- } \\
\text { staltet sein, damit es den } \\
\text { Anforderungen des Nutzer- } \\
\text { typs entspricht? In welcher } \\
\text { Situation und wie würde } \\
\text { dieser Nutzertyp das System } \\
\text { nutzen? usw. }\end{array}$ & 20 \\
\hline Pause & $\begin{array}{l}\text { Imbiss/Getränke } \\
\text { bereitstellen, an } \\
\text { Themenspeicher } \\
\text { erinnern }\end{array}$ & $\begin{array}{l}\text { Informelle Gespräche mit } \\
\text { den Teilnehmer(inne)n } \\
\text { suchen }\end{array}$ & 20 \\
\hline $\begin{array}{l}\text { Ergebnisprä- } \\
\text { sentation der } \\
\text { Kleingruppen } \\
\text { im Plenum }\end{array}$ & $\begin{array}{l}\text { Präsentation der Er- } \\
\text { gebnisse der Klein- } \\
\text { gruppen }\end{array}$ & $\begin{array}{l}\text { Was zeichnet den Nutzertyp } \\
\text { aus? Für welchen Zweck und } \\
\text { wie würde der Nutzertyp } \\
\text { das System nutzen? Was } \\
\text { wäre ihm dabei wichtig? } \\
\text { usw. }\end{array}$ & 30 \\
\hline $\begin{array}{l}\text { Abschluss/ } \\
\text { Ausblick }\end{array}$ & $\begin{array}{l}\text { Zusammenfassung } \\
\text { der Erkenntnisse } \\
\text { (inkl. Themen- } \\
\text { speicher), Ausblick } \\
\text { auf das weitere } \\
\text { Vorgehen, Bitte um } \\
\text { Feedback (anonymer } \\
\text { Feedbackfrage- } \\
\text { bogen) }\end{array}$ & $\begin{array}{l}\text { Was hat Ihnen gut gefallen? } \\
\text { Was weniger gut? Welche } \\
\text { Fragen sind offengeblieben? } \\
\text { usw. }\end{array}$ & 20 \\
\hline
\end{tabular}

\subsubsection{Workshopdokumentation}

Es ist ratsam, den Workshop mit einer Videokamera oder einem Tonband aufzuzeichnen, um die Diskussion und Interaktion sowohl aus dem Plenum als auch aus den Kleingruppen bei der anschließenden Auswertung nachvollziehen zu können. Die Daten werden nach Ablauf des Projekts gelöscht, was den Teilnehmer(inne)n so auch kommuniziert wird.

Die unterschiedlichen Visualisierungen, wie zum Beispiel die Nutzerprofile (s. Abb. 6), die im Rahmen des Workshops gemeinsam erarbeitet werden, stellen zudem eine Dokumentation der Diskussion und v. a. der in der Gruppe herausgearbeiteten Ergebnisse dar. Insbesondere in interdisziplinären Teams ist es ratsam, das Material (Skizzen, bunte Klebezettel, Artikuliertes) für alle im Projektteam gut verständlich und lebensnah aufzubereiten, um dann ausgehend davon die weitere Konzeption vorzunehmen. 


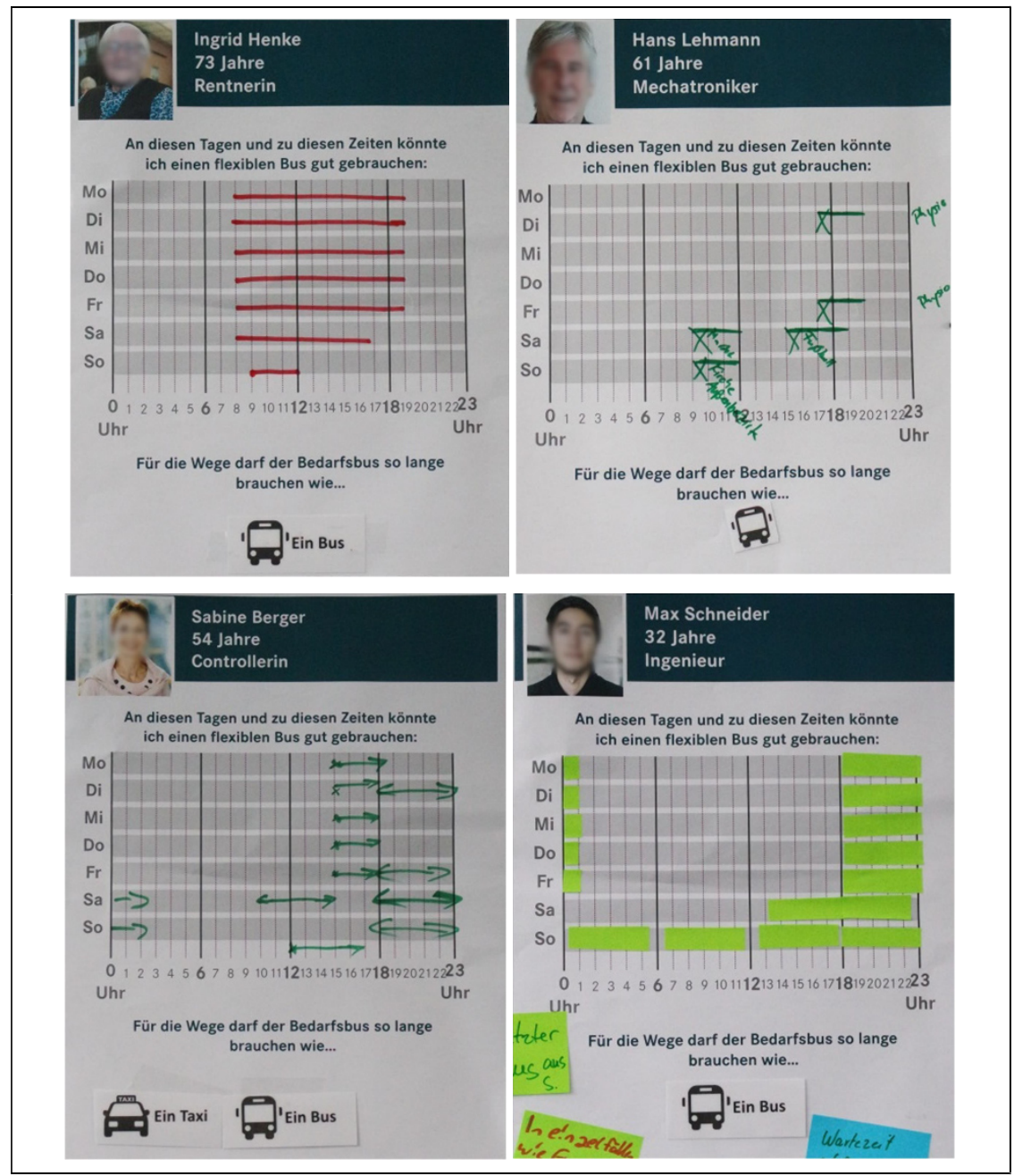

Abbildung 9: Die unterschiedlichen Präferenzen der Nutzertypen bezüglich der Betriebszeiten des Busses, kenntlich gemacht durch das Einzeichnen oder Bekleben der gewünschten Zeitfenster. (C) Salome Keller.

So lässt sich am Beispiel der im Workshop des Reallabors Schorndorf diskutierten Buseinsatzzeiten zeigen, dass Visualisierungen dabei helfen, Ergebnisse zu den bzw. Unterschiede zwischen den einzelnen Nutzertypen herauszustellen. Die visuelle Darstellung in Form von Markierungen im Wochenkalender kann als zusammenfassendes Ergebnis der Kleingruppen verstanden werden (s. Abb. 9). 


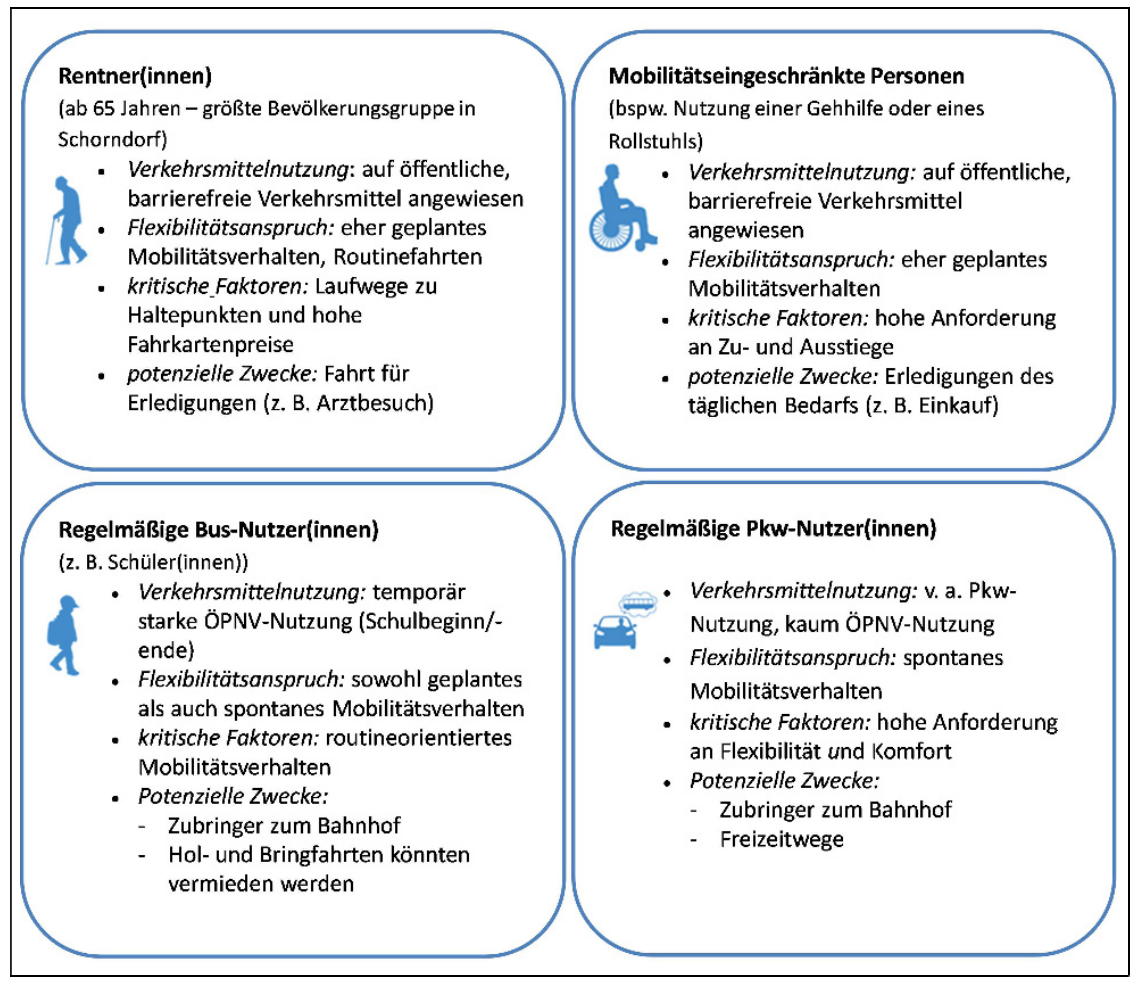

Abbildung 10: Beschreibung der identifizierten Nutzertypen anhand ausgewählter Charakteristika im Reallabor Schorndorf. (C) Laura Gebhardt, Alexandra König.

Die im Nachgang zum Workshop des Reallabors Schorndorf vorgenommenen Beschreibungen der Nutzertypen anhand ausgewählter Charakteristika (hier Verkehrsmittelnutzung, Flexibilitätsanspruch, kritische Faktoren und potenzielle Zwecke) zeigen beispielhaft, wie eine Aufbereitung der gewonnenen Ergebnisse aussehen kann (s. Abb. 10). Sie helfen dem transdisziplinären Team, ein möglichst konkretes Bild der Nutzergruppen vor Augen zu haben.

\subsubsection{Verwertung und Kommunikation der Ergebnisse}

Es empfiehlt sich, die Workshopergebnisse in Form der gemeinsam erstellten visuellen Dokumentationen sowie anhand der Beobachtungen (Protokoll, Tonund Videoaufnahmen) im Projektteam zu diskutieren und zu strukturieren. Hierbei helfen das bereits erwähnte Leitplankendokument sowie das Spezifikationsheft (s. hierzu Abschnitt 2.1). Im Leitplankendokument werden Fragen, die sich im Workshop als relevant herausgestellt haben, tabellarisch gesammelt und mit 
den Projektbeteiligten diskutiert, priorisiert und bearbeitet. Innerhalb des Projektteams beschlossene Antworten auf die offenen Fragen werden als Leitplanken festgehalten und dann in das Spezifikationsheft überführt. Das Spezifikationsheft dient der Dokumentation aller Erkenntnisse und ist letzten Endes die Grundlage für die Ausgestaltung des Systems. Zudem werden hier Argumentationsstränge und die Begründung der Parameterfestlegung festgehalten. Nach dem Workshop werden das Leitplankendokument und das Spezifikationsheft durch die Projektpartner anhand der Ergebnisse aktualisiert.

Neben der Kommunikation innerhalb des Projektteams gilt es, die Erkenntnisse des Workshops für die Teilnehmer(innen) sowie ggf. in der Öffentlichkeit transparent zu machen. Dies kann zum einen über ein Schreiben an die Teilnehmer(innen) des Workshops (inkl. einer Danksagung für die Teilnahme) geschehen, zum anderen durch das Publizieren der zentralen Erkenntnisse auf der Projekthomepage und in lokalen Medien (z. B. im örtlichen Wochenblatt).

Zwischen dem ersten und dem zweiten Workshop, der im Folgenden beschrieben wird, sollten mindestens anderthalb Monate Zeit eingeplant werden, damit die Ergebnisse des ersten Workshops in ausreichender Tiefe ausgewertet und für den anschließenden Workshop aufbereitet werden können.

\subsection{Ideen und Konzepte gemeinsam entwickeln- Co-Creation-Workshop II}

In Abhängigkeit vom Forschungsgegenstand und dessen Komplexität bietet es sich an, statt einem mehrere solcher Workshops durchzuführen, die sich jeweils einer Auswahl von Teilaspekten widmen. Um die Teilnehmer(innen) nicht zu überfordern, wird empfohlen, nicht mehr als fünf Teilaspekte je Workshop zu bearbeiten. Im Reallabor Schorndorf war ein Teilaspekt der Buchungsprozess des Bedarfsbusses, ein weiterer war das Innenraumkonzept des Bedarfsbusses. Ein wichtiges Entscheidungskriterium für die Workshopanzahl bietet also der Umfang der Teilaspekte.

\subsubsection{Workshopziele}

Ziele des Workshops sind die Entwicklung von ersten Ideen zur Ausgestaltung des zu entwickelnden Systems und die Bewertung von konkreten Konzeptideen. Zudem soll am Ende des Workshops ein Prototyp vorliegen, der das Konzept anschaulich darstellt. Dabei kann der Prototyp auch ein Ablaufschema darstellen, muss also kein haptisches Produkt sein. Wie bereits in Kapitel 1 erwähnt, kann nicht vorausgesetzt werden, dass sich die zukünftigen Nutzer(innen) ihrer eigenen Anforderungen an das zu entwickelnde System umfassend bewusst sind und diese adäquat äußern können. Mit dem Workshop II wird daher das Ziel verfolgt, die 
eher impliziten Denkweisen und Bedürfnisse der zukünftigen Nutzer(innen) zutage zu fördern und mittels Kreativtechniken in konkrete Konzeptideen und -entwürfe zu übersetzen (für eine Übersicht an Kreativtechniken, s. Degele et al. 2009). Die TraSy-Methode generiert also im Co-Creation-Workshop II mehrere Ergebnisse:

1) Entwicklung von Ideen zur konkreten Systemausgestaltung

2) Entwicklung und Bewertung von konkreten Konzeptentwürfen

3) Umsetzung der vielversprechendsten Konzeptentwürfe als Prototyp

Im Reallabor Schorndorf wurden in Phase 3 (Ideen und Konzepte gemeinsam entwickeln) Co-Creation-Workshops zur Entwicklung von Ideen und Konzeptentwürfen für den Innenraum des Bedarfsbusses sowie für den Bestell- und Buchungsprozess des Busses durchgeführt.

\subsubsection{Vorphase: Bisher identifiziertes Wissen nutzbar machen}

Die Ergebnisse der beiden vorherigen Phasen (Nutzer(innen) im alltäglichen Kontext verstehen (Abschnitt 2.1) und Nutzertypen identifizieren und beschreiben (Abschnitt 2.2), in denen die Nutzerprofile und die Szenarien erstellt wurden, werden, wie in Abb. 11 dargestellt, als Input für den Workshop verwendet. An dieser Stelle im Ablauf der TraSy-Methode enthält das Spezifikationsheft alle bisher definierten Angaben zur Charakterisierung der Nutzertypen. Zudem werden hier die Nutzungsszenarien detailliert beschrieben. Das Spezifikationsheft liefert den Rahmen für die Arbeit im Workshop, indem es die nicht mehr veränderbaren Kriterien beschreibt. Diese werden den Teilnehmer(inne)n des Workshops präsentiert, um der kreativen Arbeit einen Rahmen zu geben. Das Leitplankendokument enthält noch unbeantwortete Fragen in Bezug auf die Charakterisierung der Nutzertypen und deren spezifischen Anforderungen, die im Rahmen des Work-

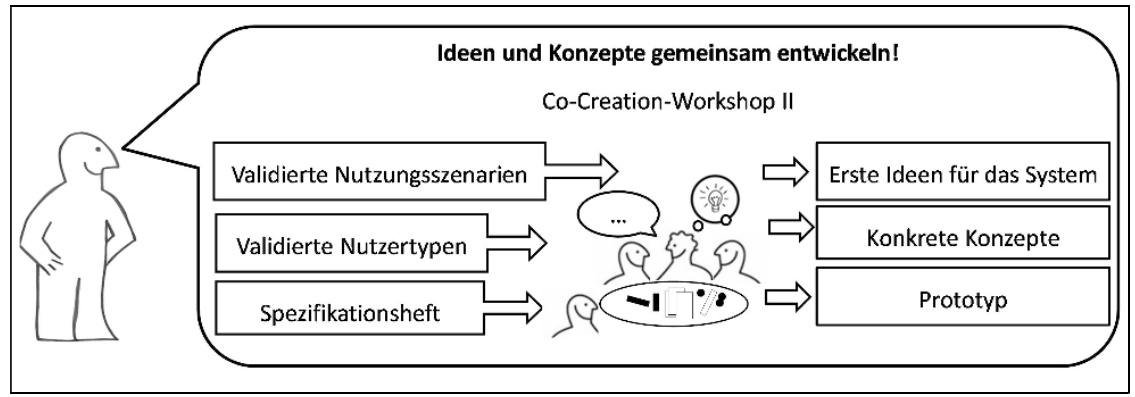

Abbildung 11: Co-Creation-Workshop II zur Entwicklung von konkreten Ideen zur Gestaltung des Systems. (C) Laura Gebhardt, Alexandra König. 
shops zu beantworten sind. Im Falle des Reallabors Schorndorf war eine solche Frage beispielsweise: "Wie können auch Nutzer(innen) des Nutzertyps ,Rentner(innen)' ohne Mobiltelefon den Bus von unterwegs buchen oder Fahrtdetails wie die Abfahrtszeit erfragen?"“

\subsubsection{Inhaltliche Vorbereitung}

Für die Durchführung des Workshops sind einige Vorarbeiten notwendig: Das Leitplankendokument enthält offene Fragen, die im Rahmen des Workshops zu adressieren sind. Aus dem Spezifikationsheft müssen jene Anforderungen an das System extrahiert werden, die für das Thema des Workshops von Relevanz sind. Diese Anforderungen sollten von den Wissenschaftler(inne)n in enger Absprache mit den Praxispartnern so aufgearbeitet werden, dass sie für die Teilnehmer(innen) des Workshops verständlich sind. Auf die Verwendung von Fachsprache sollte verzichtet werden. Wenn dies nicht möglich ist, sollten Fachbegriffe in allgemeinverständlicher Weise definiert und beschrieben werden. Die Anforderungen sind Faktoren, die im Rahmen des Vorhabens nicht beeinflussbar sind; sie ergänzen die oben erwähnten und auf die Nutzertypen und Nutzungsszenarien bezogenen Kriterien. Beide Arten von Restriktionen müssen den Workshopteilnehmer(inne)n zu Beginn des Workshops kommuniziert werden. Es empfiehlt sich, dafür eine grafische Darstellung zu nutzen, die den Teilnehmer(inne)n jederzeit während des Workshops verfügbar ist.

Zudem gilt es natürlich, das Wissen, das in Phase 1 und insbesondere in Phase 2 generiert wurde, so aufzubereiten, dass es den Workshopteilnehmer(inne)n präsentiert werden kann. Dafür müssen die im Workshop I gemeinsam mit den Teilnehmer(inne)n erarbeiteten Nutzerprofile und Nutzungsszenarien (s. Abb. 6 und 7) inhaltlich und grafisch bearbeitet werden. Die Aufbereitung der Nutzerprofile und Nutzungsszenarien übernehmen die Wissenschaftler(innen) im Team.

Des Weiteren sind für die Workshopvorbereitung dieselben Punkte in Bezug auf die Raumwahl zu beachten, die in Abschnitt 2.2.3 zur Vorbereitung des Co-Creation-Workshops I beschrieben wurden.

\subsubsection{Ansprache und Gewinnung der Teilnehmer(innen)}

Der Co-Creation-Workshop II dauert einen ganzen Tag. Dies macht die Gewinnung von Teilnehmer(inne)n nicht einfach. Daher ist ein frühzeitiger Rekrutierungsstart wichtig. Am Co-Creation-Workshop II können dieselben Personen teilnehmen wie am Co-Creation-Workshop I, die Gruppe der teilnehmenden Personen kann aber auch ganz oder in Teilen anders sein. Für die Rekrutierung der Teilnehmer(innen) gilt dasselbe wie für den Co-Creation-Workshop I (s. Abschnitt 2.2.4). Ziel sollte es sein, eine Teilnehmerzahl von mindestens 12 Perso- 
nen zu erreichen, so dass vier Kleingruppen mit mindestens je drei Teilnehmer(inne)n gebildet werden können.

Die Einladung der Teilnehmer(innen) erfolgt schriftlich mehrere Wochen vor dem Workshop. Die Einladung enthält neben Angaben zu Ort, Zeit und Dauer des Workshops auch eine kurze und verständliche Beschreibung der Ziele und des groben Workshopablaufs.

\subsubsection{Gruppeneinteilung und Moderation}

Auch im Co-Creation-Workshop II gibt es neben Diskussionsphasen in der Gesamtgruppe Phasen der Kleingruppenarbeit. Jede Kleingruppe setzt sich aus drei bis sechs Teilnehmer(inne)n zusammen. Die Kleingruppen können, im Unterschied zum Workshop I, aus zufällig ausgewählten Teilnehmer(inne)n des Workshops gebildet werden, da die persönliche Zugehörigkeit zu einem der Nutzertypen nicht erforderlich ist. Zur zufälligen Gruppeneinteilung kann beispielsweise eine Packung von Celebrations-Süßigkeiten genutzt werden. Durch das Ziehen der verschiedenen Süßigkeiten wird die Zuordnung der Teilnehmer(innen) zu den Gruppen bestimmt. Auch diesmal ist in jeder der Kleingruppen ein(e) Wissenschaftler(in) aus dem Projektteam sowie ein(e) Moderator(in) anwesend. Falls im Projektteam keine Mitglieder vorhanden sind, die über Moderationskompetenzen verfügen, so dass externe Moderator(inn)en engagiert werden, müssen diese mit dem Thema so vertraut sein, dass sie die Gruppenarbeit auch inhaltlich gut unterstützen können, und dafür ggf. durch die beteiligten Wissenschaftler(innen) entsprechend vorbereitet werden.

\subsubsection{Durchführung des Workshops}

Der Workshop dauert ungefähr acht Arbeitsstunden. Eine beispielhafte Agenda des Workshops ist in Tab. 2 zu sehen, die neben dem zeitlichen und inhaltlichen Workshopablauf auch zeigt, welche Agendapunkte im Plenum und welche in der Gruppe stattfinden. Um die Einhaltung der straffen Agenda sicherzustellen, sind mehrere Rahmenbedingungen zu beachten: Es hat sich gezeigt, dass vor allem eine gute Vorbereitung des Arbeitsmaterials von großer Bedeutung für eine flüssige Durchführung des Workshops ist. Zudem hat sich erwiesen, dass es für die Einhaltung der Agenda wichtig ist, dass die Teilnehmer(innen) gut auf die Arbeit im Workshop eingestimmt werden. Je mehr Zeit am Anfang für die Einführung eingeplant wird, desto weniger Fragen und Schwierigkeiten ergeben sich während des Prozesses. Für die Einhaltung der Agenda ist zudem die Rolle der Moderation hervorzuheben, auf die im Weiteren genauer eingegangen wird.

$\mathrm{Zu}$ Beginn des Workshops ist eine Vorstellungsrunde essenziell, um eine angenehme und vertraute Atmosphäre zwischen den Teilnehmer(inne)n zu schaffen. 
Tabelle 2: Agenda des Co-Creation-Workshops II (Fassung für das Projektteam).

\begin{tabular}{|l|l|}
\hline \multicolumn{2}{|l|}{ Agenda des Co-Creation-Workshops II } \\
\hline 08:30 & Begrüßung (Plenum) \\
09:40 & Vorstellungsrunde (Plenum) \\
09:40 & Einführung, Leitplankenvorstellung und Kleingruppeneinteilung (Plenum) \\
10:35 & Präsentation der Nutzerprofile und der Nutzungsszenarien (Kleingruppe) \\
$10: 50$ & Formulierung des systembezogenen Teamauftrags (Kleingruppe) \\
$11: 15$ & Generierung von Ideen und konkreten Konzepten (Kleingruppe) \\
$12: 00$ & Mittagspause \\
$13: 15$ & Erstellen von Prototypen (Kleingruppe) \\
$14: 20$ & Vorbereitung der Prototypenpräsentation (Kleingruppe) \\
$14: 40$ & Kaffeepause \\
$14: 55$ & Präsentation der Prototypen (Plenum) \\
$15: 55$ & Bewertung der Restriktionen (Plenum) \\
$16: 25$ & Zusammenfassung und Abschluss (Plenum) \\
\hline
\end{tabular}

Um bereits in dieser frühen Workshopphase eine kreative Stimmung zu erzeugen, eignet sich eine spielerische Selbstpräsentation mithilfe von Metaphern. Der Arbeitsauftrag könnte lauten: „Nennen Sie ein Tier oder eine Pflanze, mit dem/ der Sie sich identifizieren, und beschreiben Sie in zwei bis drei Sätzen, warum Sie dieses Tier oder diese Pflanze gewählt haben." Hierbei geht es vor allem darum, dass die häufig zu bemerkende anfängliche Zurückhaltung der Teilnehmer(innen) überwunden wird.

Um die Teilnehmer(innen) zu Beginn auf das Workshopthema einzustimmen, werden sie durch die anwesenden Vertreter(innen) des Projektteams über die Projektziele, die bisherigen Ergebnisse und die weiteren Schritte informiert. Zudem ist es wichtig, den Teilnehmer(inne)n die Workshopziele so vorzustellen, dass möglichst eine einheitliche Erwartungshaltung entsteht. Auf die Ziele und den groben Ablauf des Workshops einzugehen, ist auch dann sinnvoll, wenn diese schon in der schriftlichen Einladung aufgeführt wurden. Erfahrungsgemäß sind mindestens 15 Minuten für die Vermittlung der Zielstellung und der methodischen Herangehensweise des Workshops einzuplanen. 


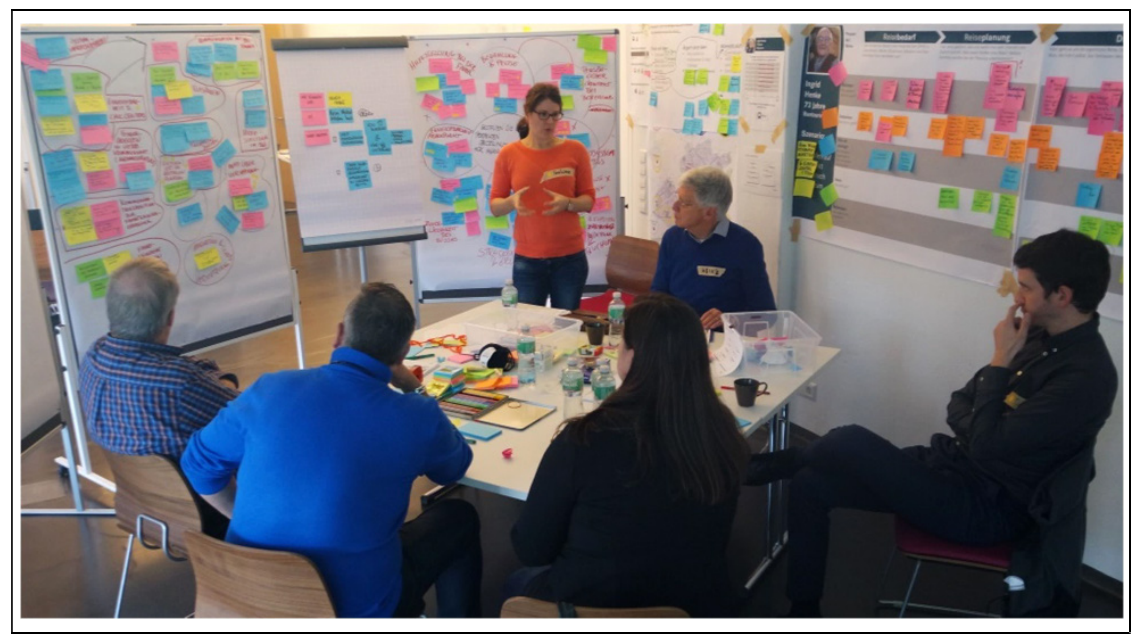

Abbildung 12: Kleingruppenarbeit innerhalb des Co-Creation-Workshops II in der Ideengenerierungsphase. Die bisherigen Ergebnisse sollten, wie hier in Form der Flipcharts und Metaplanwände, stets für alle sichtbar sein. (C) Salome Keller.

Schließlich werden im Rahmen der Einführung im Plenum die inhaltlichen Restriktionen vorgestellt, die im Workshop zu beachten sind (s. Abschnitt 2.3.3). Jede Restriktion, die eine Rahmenbedingung für das System beschreibt, wird möglichst präzise erklärt. Die Teilnehmer(innen) sollten ermutigt werden, Nachfragen zu formulieren, falls Unklarheiten bestehen, denn es ist wichtig, dass jede(r) Teilnehmer(in) die Rahmenbedingungen des Systems kennt und versteht.

Die eigentliche kreative Arbeit innerhalb des Workshops findet in den Kleingruppen statt. Hierfür sollten geeignete Räume vorhanden sein, um die einzelnen Kleingruppen voneinander zu trennen und die genügend Platz bieten, so dass jede Kleingruppe sowohl eigene Ergebnisse auf ausreichend vielen Pinnwänden festhalten kann, als auch von den Visualisierungen der bisherigen Ergebnisse ,umgeben' sein kann (s. Abb. 12). Ein(e) mit dem Thema vertraute(r) Moderator(in) (bestenfalls aus dem Projektteam stammend) kontrolliert den zeitlichen Rahmen der Teamarbeit, steht den Teammitgliedern für Nachfragen zur Seite und regt, wenn nötig, die Kreativität der Teilnehmer(innen) durch gezielte Nachfragen an. Jedoch sollte die Moderation möglichst wenig in die Selbstorganisation der Gruppe eingreifen. Konkret bedeutet dies, dass der bzw. die Moderator(in) den Workshopteilnehmer(inne)n nicht das Gefühl vermitteln darf, er/sie fühle sich aufgrund der eigenen wissenschaftlichen Qualifikationen überlegen. Zudem muss die Moderation sich zurückhaltend und neutral verhalten, also die Einschätzungen der Teilnehmer(innen) nicht bewerten. Für die Gruppenarbeit sollten einige Grund- 
regeln festgelegt werden, die das Verhalten untereinander festlegen. Sie sollten möglichst zu Beginn der Gruppenarbeit an alle Teilnehmer(innen) kommuniziert werden. Als besonders relevant werden die folgenden Regeln erachtet: Wir lassen einander aussprechen! Alle Ideen sind wertvoll! Lassen Sie auch unkonventionelle Gedanken zu! Bewerten Sie nicht die Ideen der anderen Teilnehmer(innen)! Bauen Sie auf den Ideen der anderen Teilnehmer(innen) auf und ,spinnen' Sie den Faden weiter!

Der Workshop baut auf den in Phase 2 erarbeiteten Nutzertypen und Nutzungsszenarien auf. Jede Kleingruppe widmet sich dabei einem Nutzertyp. Die Nutzerprofile und Nutzungsszenarien werden den Teilnehmer(inne)n durch die Moderation anhand der Steckbriefe und Szenarien präsentiert, die in Phase 2 erarbeitet und in Vorbereitung auf den Workshop von den Wissenschaftler(inne)n aufbereitet wurden (s. Abschnitt 2.3.3). Um sich noch besser in den Nutzertyp hineinversetzen zu können, überlegt sich jede Kleingruppe unter Anleitung der Moderation Ziele und Motive des ihr zugewiesenen Nutzertyps, zum Beispiel anhand vorbereiteter Fragen (s. Abb. 13 links), die sich einem Teilaspekt widmen und möglichst konkret zu formulieren sind. Erfahrungsgemäß regt die Arbeit mit Fragen die Diskussion innerhalb der Kleingruppe an und erzeugt konkrete Antworten.

Nach einer Kaffeepause, die zugleich die Möglichkeit zum weiteren Austausch innerhalb und zwischen den Kleingruppen bietet, kommen die Kleingruppen erneut zusammen. Nun wird der auf das System bezogene Gruppenauftrag formuliert. Dieser geht von dem der Gruppe zugewiesenen Nutzerprofil und Nutzungsszenario aus: „Entwerfen Sie ein System Z, das so gestaltet ist, dass Nutzertyp X seine Ziele $Y_{1}, Y_{2}, \ldots$ erreicht.“ Der Auftrag sollte möglichst präzise formuliert und für alle sichtbar notiert werden.

Die Teilnehmer(innen) werden also auf der Grundlage der Ziele und Motive der Nutzertypen aufgefordert, ein System zu entwickeln, das den Nutzertyp bei der Zielerreichung und der Bedürfnisbefriedigung unterstützt. In einem ersten Schritt werden die Ideen aller Gruppenmitglieder eingeholt. Diese werden alle aufgeschrieben und angeheftet. Hierbei gilt das Prinzip, dass möglichst viele unterschiedliche Ideen gesammelt werden sollen. Die Arbeit mit selbstklebenden Postits, wie auf Abb. 13 rechts zu sehen, bietet sich an, um individuelle Ideen aufzuschreiben, den Teilnehmer(inne)n die Angst vor dem weißen Blatt zu nehmen und Ideen anschließend flexibel umsortieren zu können. Wenn keine neuen Ideen mehr genannt werden, werden die gesammelten Ideen in einem zweiten Schritt durch die Teilnehmer(innen) zuerst geclustert und dann bewertet. Für die Bewertung eignen sich Klebepunkte - zum Beispiel jeweils drei pro Teilnehmer(in) -, die die Abstimmungsergebnisse visualisieren und quantifizieren. Der freien Ideengenerierung folgt dann in einem dritten Schritt die Formulierung konkreter 


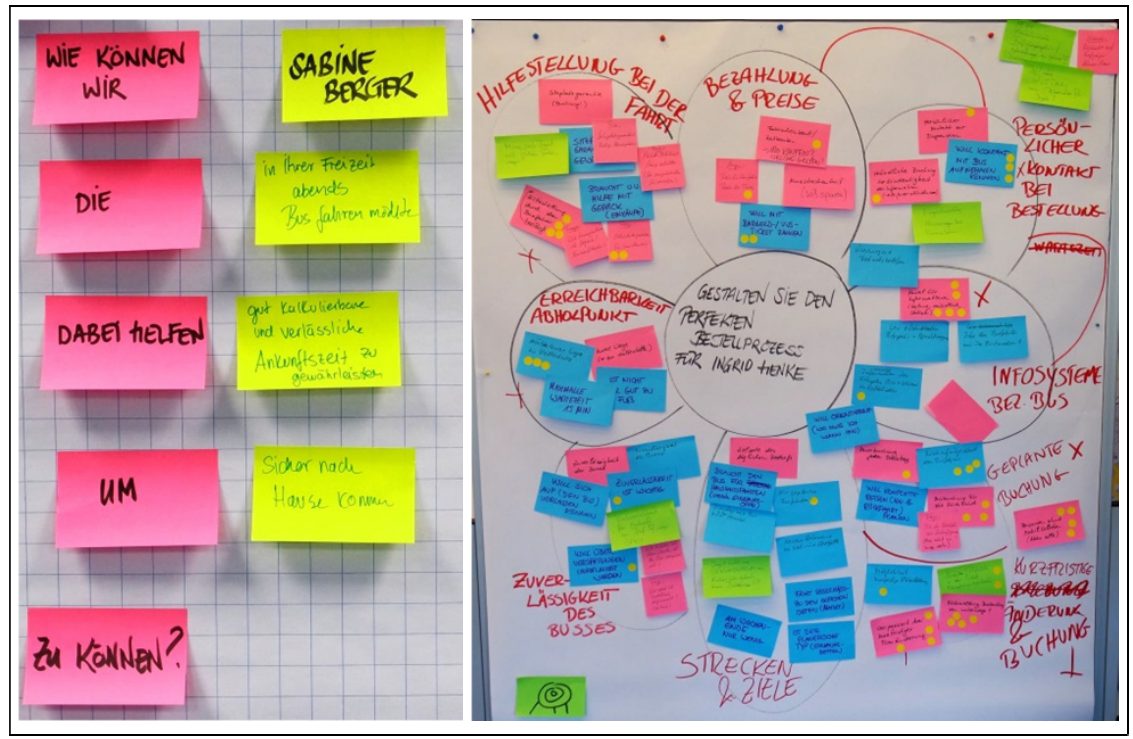

Abbildung 13: Links: Mithilfe einer vorgegebenen Frage werden die Bedürfnisse eines Nutzertyps (hier: Sabine Berger) in konkrete Anforderungen übersetzt. Rechts: Darstellung der Ergebnisse der Kleingruppenarbeit (hier die Cluster als Blütenblätter dargestellt) und Bewertung der Ideen durch die Teilnehmer(innen) mithilfe von gelben Klebepunkten. (C) Salome Keller.

Konzepte anhand der am besten bewerteten Ideen. Diese Konzepte beschreiben einen Teilaspekt des zu entwickelnden Systems möglichst konkret. Im Reallabor Schorndorf war das beispielsweise der Bezahlungsprozess auf einer Fahrtenbuchungswebseite. Die Moderation stellt sicher, dass ideale Konzepte beschrieben werden, die möglichst alle Anforderungen des Nutzertyps an das System erfüllen.

Nachdem in der Kleingruppe zuerst Ideen und dann Konzepte entwickelt wurden, ist die Übersetzung des vielversprechendsten Konzepts in einen Prototyp die nächste Aufgabe der Kleingruppe (s. Abb. 14 links). Für die Auswahl bietet sich wiederum die Priorisierung und Abstimmung mithilfe von Klebepunkten an. Für die Erstellung des Prototyps sollten den Teilnehmer(inne)n möglichst viele Materialien, wie verschiedenfarbige Stifte, LEGO ${ }^{\circledR}$-Figuren und -Bausteine, Papier, Knetmasse etc. zur Verfügung gestellt werden, um die plastische Umsetzung zu ermöglichen. Die Teilnehmer(innen) sollten auch angeregt werden, ihre Visionen grafisch in kleinen Skizzen zu visualisieren. Die plastische und grafische Darstel- 


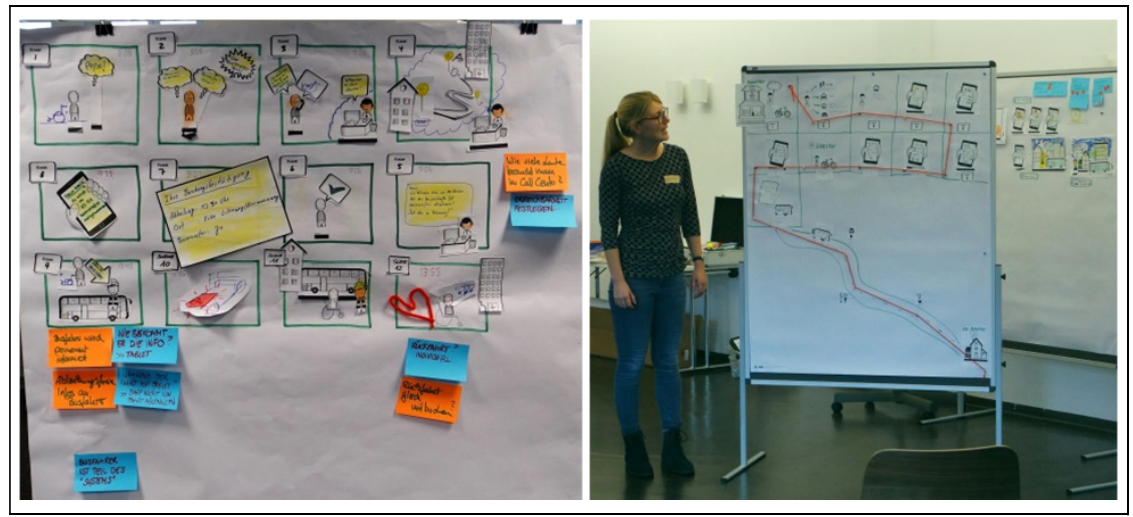

Abbildung 14: Links: Das Ergebnis der Kleingruppenarbeit, der Prototyp, muss kein anfassbares Produkt sein, sondern kann, wie hier, auch die Form eines Ablaufdiagramms haben. Wichtig ist die plastische und grafische Aufbereitung der Ideen, die eine Diskussion anregen soll. Rechts: Ein wichtiger methodischer Baustein des Workshops ist die Präsentation der Ergebnisse aus den Gruppenarbeiten im Plenum. C Salome Keller.

lung der Ergebnisse ermöglicht zum Ersten die Diskussion innerhalb der Kleingruppe und veranschaulicht zum Zweiten die Ergebnisse für die anschließende Präsentation im Plenum. Das Präsentieren der Ergebnisse der Kleingruppenarbeit im Plenum stellt ein wichtiges methodisches Element dar, weil es dazu zwingt, Argumentationsstränge zu artikulieren, und erlaubt, mit den anderen Workshopteilnehmer(inne)n über offene Fragen und potenzielle Herausforderungen zu diskutieren.

Für die Präsentation der Ergebnisse aus der Kleingruppenarbeit kommen alle Teilnehmer(innen) des Workshops wieder im Plenum zusammen. Nacheinander stellen sich die Gruppen gegenseitig ihre Ergebnisse anhand des angefertigten Prototyps vor (s. Abb. 14 rechts). Alle Teilnehmenden werden durch die Moderation ausdrücklich aufgefordert und ermuntert, Fragen zu stellen sowie Ideen und Anmerkungen zum Prototyp zu äußern. Weiterhin werden nach jeder einzelnen Präsentation die Grenzen des Prototyps besprochen. Hierbei können Fragen wie diese leiten: „Warum kann das System X nicht Y?“ (wobei Y eine Nutzeranforderung sein könnte, die sich im vorherigen Workshop und in der Arbeit mit den Nutzertypen als relevant herausgestellt hatte), „Unter welchen Umständen könnte es beim Einsatz oder der Nutzung des Systems X Schwierigkeiten geben?" usw.

Der Workshoptag schließt mit einer Zusammenfassung der Ergebnisse und einem Ausblick auf die nächsten Schritte innerhalb des Projekts durch die Moderation. 


\subsubsection{Workshopdokumentation}

Wie bereits in Bezug auf Workshop I beschrieben (s. Abschnitt 2.2.7), ist es empfehlenswert, bereits im Rahmen der Ansprache und Gewinnung der Teilnehmer(innen) zu kommunizieren, dass es Bild- und Tonaufnahmen geben wird, die lediglich für interne Zwecke verwendet werden. Innerhalb des Workshops wird dies den Teilnehmer(inne)n noch einmal schriftlich vorgelegt, mit der Bitte, sich durch ihre Unterschrift damit einverstanden zu erklären.

Die Video- oder Audioaufnahmen werden erstellt, damit die Argumentationsstränge in den Diskussionen im Nachhinein nachvollzogen werden können, ohne dass alles während des gesamten Workshops schriftlich protokolliert werden muss. Sämtliche Ergebnisse der Kleingruppenarbeit (z. B. Notizen auf den Flipcharts, alle Post-its) sind abzufotografieren. Natürlich sind auch alle erarbeiteten Visualisierungen, wie zum Beispiel die Prototypen, die im Rahmen des Workshops gemeinsam erarbeitet werden, Teil der Dokumentation der Ergebnisse.

Weiterhin ist es die Aufgabe der Moderation, während der Kleingruppenarbeit und den Diskussionen im Plenum in einem Protokoll festzuhalten, wenn es zu Problemen, Verzögerungen oder Schwierigkeiten bei der Durchführung eines der Schritte oder zu Konflikten zwischen den Teilnehmer(inne)n kommt.

\subsubsection{Verwertung und Kommunikation der Ergebnisse}

Im Anschluss an den Workshop werden die Ergebnisse innerhalb des Projektteams diskutiert, aufgearbeitet und ausgewertet. Allen Projektteammitgliedern muss Zugang zu den Workshopergebnissen verschafft werden, damit sich auch diejenigen, die nicht am Workshop teilgenommen haben, einen Überblick über den Verlauf und die Ergebnisse erarbeiten können. Die Auswertung der Workshopergebnisse wird zwischen den wissenschaftlichen Projektteammitgliedern aufgeteilt, und zwar so, dass jede(r) einen Teil der Auswertung übernimmt, bevor diese dann zu einem Gesamtergebnis zusammengefügt werden. Die Ergebnisse sind dabei möglichst umfangreich grafisch aufzuarbeiten und zu strukturieren. Wichtig ist, dass nicht nur eine sachlich-thematische Auswertung erfolgt (,X Workshopteilnehmer(innen) haben Bedenken geäußert, dass Nutzer(innen) bei der Nutzung des Prototyps Y Schwierigkeiten mit Z haben könnten"), sondern auch eine Auswertung der Argumentationsstränge. Dies ist wichtig für die spätere Nachvollziehbarkeit und Transparenz der Ergebnisse.

Die im Projektteam diskutierten, sich auf offene Fragen beziehenden Ergebnisse werden in das Leitplankendokument aufgenommen. Fragen, die vor dem Workshop noch nicht beantwortet werden konnten, können im Idealfall anhand der Workshopergebnisse nun beantwortet werden. Wenn sich auf Basis des Workshops neue Fragen ergeben haben, werden diese auch in das Leitplankendokument aufgenommen. Die Anforderungen an das System, die von den Projektbetei- 
ligten ,abgesegnet" werden, werden in das Spezifikationsheft übernommen. In diesem werden auch die Argumentationsstränge und Begründungen der Parameterfestlegung festgehalten. Mit der Aktualisierung des Leitplankendokuments und des Spezifikationshefts ist die Auswertung des Co-Creation-Workshops II abgeschlossen.

Anschließend gilt es, wie schon beim Workshop I, die Ergebnisse des Workshops den Workshopteilnehmer(inne)n sowie der interessierten Öffentlichkeit zu kommunizieren, zum einen in Form eines Schreibens an die Teilnehmer(innen) des Workshops (inkl. eines schriftlichen Dankeschöns für die Teilnahme), zum anderen durch die Publikation zentraler Ergebnisse auf der Projekthomepage und in lokalen Medien (z. B. im örtlichen Wochenblatt).

Im Anschluss an die hier präsentierten Phasen der TraSy-Methode muss im weiteren Projektverlauf die Erprobung des entwickelten Systems im Realkontext stattfinden (s. Abb. 1). Hierfür ist ein mehrmonatiger Pilotbetrieb des Systems vorzusehen, der weitere Erkenntnisse zur Nutzerfreundlichkeit des entwickelten Systems liefert. Die Ergebnisse aus qualitativen und quantitativen Nutzerbefragungen sollten dann wiederum ins Leitplankendokument und ins Spezifikationsheft einfließen. Im Reallabor Schorndorf wurde ein Pilotbetrieb des Bedarfsbusses von März bis Dezember 2018 durchgeführt. Dabei wurde das System durch die Nutzer(innen) getestet, evaluiert und kontinuierlich verbessert. Für detaillierte Angaben dazu siehe Brost et al. (2018).

\section{Reflexion und Empfehlungen zum Einsatz der TraSy-Methode}

In diesem Beitrag wurde eine Methode zur transdisziplinären Entwicklung von soziotechnischen Systemen vorgestellt, d. h. von Systemen, die das alltägliche Handeln von Menschen betreffen, also in konkrete gesellschaftliche Kontexte eingebettet sind. Das Zusammenführen von Ziel- und Systemwissen von Praxisakteuren aus der Zivilgesellschaft ermöglicht die bedarfsgerechte Entwicklung eines solchen Systems.

Die Anwendung der Methode sowie damit verbundene Kommunikationsprozesse sollten immer auf die Gegebenheiten vor Ort bezogen werden, was bedeutet, dass entsprechende Anpassungen im Vorgehen vorgenommen werden müssen.

Im Rahmen der Entwicklung und Anwendung der TraSy-Methode im Reallabor Schorndorf haben sich folgende Herausforderungen an die TraSy-Methode gezeigt, die es bereits bei der Planung von Projekten dieser Art sowie kontinuierlich im Projektverlauf zu reflektieren gilt. 


\subsection{Erwartungshaltungen der Praxispartner}

Die Erwartungshaltung aller Akteure zu Projektbeginn stellt eine wichtige Determinante für den weiteren Projektverlauf und dessen Bewertung dar. Im Sinne der geforderten „Transparenz nach innen“ bei transdisziplinären und transformativen Ansätzen (Quint et al. 2018, S. 71) wird empfohlen, der gemeinsamen Entwicklung von Zielsetzungen und Vorgehensweisen zu Beginn des Projekts ausreichend Beachtung zu schenken. Bei der Umsetzung der TraSy-Methode ist es deshalb entscheidend, frühzeitig zu kommunizieren, an welchen Stellen die Praxispartner Einfluss auf das Projekt nehmen können, um keine falsche Erwartungshaltung aufzubauen (Brandies et al. 2017). Wie von Seebacher et al. (2018) gefordert, darf aus dem „Partizipationskontinuum“ (Meyer-Soylu et al. 2016) ,[...] keine Art ,Pflicht zu andauernder Partizipation“ aller Akteure bei allen Themen und Aktivitäten abgeleitet werden" (Seebacher et al. 2018, S. 103). Bei der Durchführung der TraSy-Methode ist das ,[...] Abwägen zwischen Interessen der Praxispartner sowie forschungsbezogenen Zielen, mit Erwartungen an schnelle praxisnahe und öffentlichkeitswirksame Ergebnisse einerseits und möglicherweise als langsam wahrgenommene Produktion von Forschungserkenntnissen andererseits, hierbei häufig eine zentrale Herausforderung" (Brandies et al. 2017, S. 13).

\subsection{Die interdisziplinäre Verständigung und Einigung}

Die Entwicklung eines soziotechnischen Systems stellt besondere Herausforderungen an die Entwicklung einer gemeinsamen, disziplinübergreifenden Sprache. So hatten beispielsweise im Reallabor Schorndorf die beteiligten Psycholog(inn)en und Verkehrsingenieur(inn)e(n) ein unterschiedliches Bild vom Begriff Bedienkonzept. Die kontinuierliche gemeinsame Arbeit am Spezifikationsheft (s. Kapitel 2), in dem zentrale Termini definiert und festgehalten werden, kann dabei unterstützen, eine gemeinsame Sprache zu finden. Zudem dient das Spezifikationsheft dazu, Zielsetzungen an geeigneter Stelle zu fixieren, um der Dynamik des interdisziplinären Arbeitens zu begegnen.

Der Austausch und die Reflexion innerhalb des interdisziplinären Projektteams sind von großer Bedeutung für den Erfolg der TraSy-Methode. Eine Herausforderung dabei ist es, einerseits durch kontinuierliche Abstimmungen redundante Arbeiten, Verantwortungsdiffusion und Informationsmängel zu vermeiden und andererseits schnelle und tragfähige Entscheidungen zu treffen (Brandies et al. 2017). Diese Herausforderung wiederum kann mit der Erstellung und kontinuierlichen Pflege des Spezifikationsheftes begegnet werden, das als eine Art ,Leitwerk' die Zusammenarbeit im Projektteam strukturiert. 
Ein relevanter Aspekt für die Umsetzung der TraSy-Methode ist die Wertschätzung der Methoden und der Denkschulen der zu beteiligenden Disziplinen. Diesen liegen sehr verschiedene Methodologien zugrunde, da zwingend sozialwissenschaftliche und ,technische' Wissenschaftskulturen aufeinandertreffen. Das Spezifikationsheft kann auch helfen, dieser Herausforderung zu begegnen. Als eine Plattform zum gemeinsamen Wissensaustausch dient es der Übersetzung qualitativer Ergebnisse aus den sozialwissenschaftlichen Elementen der TraSyMethode in Anforderungen an das System. Das Spezifikationsheft stellt damit ein wichtiges Instrument zur Synthesebildung dar.

\section{Dank}

Die Autorinnen danken Svenja Kück und Christian Schmutz für die Diskussionen über eine frühere Textfassung. Insbesondere möchten sie Christina West und Belen Zevallos sowie zwei anonymen Gutachter(inne)n für die konkreten Hinweise zur Verbesserung des Textes im Rahmen des internen und externen Reviews danken. Besonderer Dank gebührt Marcel Bednarz, Christian Borowski, Dijana Hammans, Salome Keller, Christian Kuhn, Martha Miosga und Christina Zissaki für ihre Unterstützung in der Planung und Durchführung der Co-CreationWorkshops. Schließlich danken die Autorinnen den beiden Herausgebenden, Rico Defila und Antonietta Di Giulio, für ihre Rückmeldungen zum Text.

\section{Literatur}

Achtziger, A., \& Gollwitzer, P. M. (2009). Rubikonmodell der Handlungsphase [Rubicon model of action phases]. In V. Brandstätter \& J. H. Otto (Hrsg.), Handbuch der Allgemeinen Psychologie: Motivation und Emotion (S. 150-156). Göttingen: Hogrefe.

Bergold, J., \& Thomas, S. (2010). Partizipative Forschung. In G. Mey \& K. Mruck (Hrsg.), Handbuch Qualitative Forschung in der Psychologie (S. 333-344). Wiesbaden: Springer VS.

Bergold, J., \& Thomas, S. (2012). Partizipative Forschungsmethoden: Ein methodischer Ansatz in Bewegung. Forum Qualitative Sozialforschung/Forum: Qualitative Social Research, 13 (1). doi: 10.17169/fqs-13.1.1801.

Brandies, A., König, A., Viergutz, K. K., Fraedrich, E., Gebhardt, L., Ulmer, F., Sippel, T., \& Dotzauer, M. (2017). Transdisziplinäre Mobilitätsforschung unter Verwendung von Reallaboren: Integration von Stakeholderbedürfnissen und -anforderungen in die Entwicklung von Systemen bedarfsorientiert und vollautomatisiert fahrender Quartiersbusse. Vorgestellt auf der Konferenz: AAET 2017: Automatisiertes \& Vernetztes Fahren 2017, Braunschweig. https://elib.dlr.de/106113/. Zugegriffen am 01.03.2019. 
Brost, M., Gebhardt, L., Steiner, T., Neidhardt, E., Brandies, A., Karnahl, K., \& Ademeit, A.-M. (2018). Results from the real-world-laboratory based pilot operation of a demand responsive bus system. Vorgestellt auf der European Transport Conference (ETC) 2018, 10.-12.10.2018, Dublin, Irland. https://elib.dlr.de/123300/. Zugegriffen am 01.03.2019.

Büscher, C., \& Schippl, J. (2013). Die Transformation der Energieversorgung: Einheit und Differenz soziotechnischer Systeme. Technologiefolgenabschätzung - Theorie und Praxis, 22 (2), (S. 11-19).

CASS \& ProClim- (1997). Forschung zu Nachhaltigkeit und Globalem Wandel - Wissenschaftspolitische Visionen der Schweizer Forschenden. Bern: ProClim-/SANW. https:// naturwissenschaften.ch/service/publications/75640-visionen-der-forschenden. Zugegriffen am 22.02.2019.

Defila, R., \& Di Giulio, A. (2018a). Partizipative Wissenserzeugung und Wissenschaftlichkeit - ein methodologischer Beitrag. In R. Defila \& A. Di Giulio (Hrsg.), Transdisziplinär und transformativ forschen. Eine Methodensammlung (S. 39-67). Wiesbaden: Springer VS. doi: 10.1007/978-3-658-21530-9_2.

Defila, R., \& Di Giulio, A. (2018b). Reallabore als Quelle für die Methodik transdisziplinären und transformativen Forschens - eine Einführung. In R. Defila \& A. Di Giulio (Hrsg.), Transdisziplinär und transformativ forschen. Eine Methodensammlung (S. 9-35). Wiesbaden: Springer VS. doi: 10.1007/978-3-658-21530-9_1.

Degele, N., Kesselhut, K., \& Schneickert, C. (2009). Sehen und Sprechen: zum Einsatz von Bildern bei Gruppendiskussionen. Zeitschrift für Qualitative Forschung, 10 (2), (S. 363-379).

Gebhardt, L., Brost, M., \& Steiner, T. (2019). Bus on demand - ein Mobilitätskonzept mit Zukunft. Das Reallabor Schorndorf zieht nach dem Pilotbetrieb Bilanz. GAIA, 28 (1), (S. 66-68).

Gebhardt, L., Klemme, M., \& Wiegandt, C.-C. (2014). Bürgerbeteiligung und Bürgerengagement in Zeiten der Digitalmoderne - drei Thesen. disP - The Planning Review, 50 (3), (S. 11-120). doi: 10.1080/02513625.2014.979050.

Girtler, R. (2002). Methoden der Feldforschung. Wien: Böhlau.

Grosvenor, T. (2000). Qualitative Research in the Transport Sector. Vorgestellt auf der Konferenz: Transport Surveys: Raising the Standard, Grainau (24.-30.05.1997).

Hennen, L. (2012). Why do we still need participatory technology assessment?. Poiesis \& Praxis, 9 (1-2), (S. 27-41). doi: 10.1007/s10202-012-0122-5.

Hunecke, M. (2015). Mobilitätsverhalten verstehen und verändern. Psychologische Beiträge zur interdisziplinären Mobilitätsforschung. Wiesbaden: Springer VS.

Hügli, A., \& Lübcke, P. (1991). Philosophielexikon. Reinbek bei Hamburg: Rowohlt.

Jaeger-Erben, M., Nagy, E., Schäfer, M., Süßbauer, E., \& Zscheischler, J. (2018). Von der Programmatik zur Praxis: Plädoyer für eine Grounded Theory transformationsorientierter Forschung. GAIA, 27 (1), (S. 117-121). doi: 10.14512/gaia.27.1.5. 
Joss, S., \& Bellucci, S. (2002). Participatory Technology Assessment in Europe: Introducing the EUROPTA Research Project. In S. Joss \& S. Bellucci (Hrsg.), Participatory Technology Assessment: European Perspectives (S. 3-14). London: Center for the Study of Democracy.

Klötzke, M., Brost, M., Fraedrich, E., Gebhardt, L., Karnahl, K., Kopp, G., König, A., Ademeit, A., Müller, A., Sippel, T., \& Ulmer, F. (2018). Reallabor Schorndorf. Bürgernahe Entwicklung eines haltestellenlosen Quartiersbussystems. In H. Proff \& T. Fojcik (Hrsg.), Mobilität und digitale Transformation (S. 295-309). Wiesbaden: Springer Gabler. doi: 10.1007/978-3-658-20779-3_18.

König, A., Karnahl, K., Gebhardt, L., \& Klötzke, M. (2018). Reallabor Schorndorf - Bedarfsgesteuerte Mobilität gemeinsam gestalten. Vorgestellt auf der Konferenz: 12. Deutscher Nahverkehrstag, Koblenz (24.-26.04.2018) https://elib.dlr.de/120468/. Zugegriffen am 01.03.2019.

Kucharski, A., \& Merkel, S. (2018). Partizipative Technikentwicklung von Gerontotechnologie: Ansätze für mehr Akzeptanz in der Zielgruppe. Forschung Aktuell, Institut Arbeit und Technik (IAT), 6/2018.

Lösch, A. (2012). Techniksoziologie. In S. Massen, M. Kaiser, M. Reinhart \& B. Sutter (Hrsg.), Handbuch Wissenschaftssoziologie (S. 251-264). Wiesbaden: Springer VS.

Lüders, C. (2003). Teilnehmende Beobachtung. In R. Bohnsack, W. Marotzki, \& M. Meuser (Hrsg.), Hauptbegriffe Qualitativer Sozialforschung (S. 151-153). Opladen: VS Verlag für Sozialwissenschaften.

Luhmann, N. (1984). Soziale Systeme: Grundriß einer allgemeinen Theorie. Frankfurt a. M.: Suhrkamp.

Luhmann, N. (1997). Die Gesellschaft der Gesellschaft. Frankfurt a. M.: Suhrkamp.

Mayas, C., Hörold, S., \& Krömker, H. (2012). Meeting the Challenges of Individual Passenger Information with Personas. In N. Stantin (Hrsg.), Advances in Human Aspects of Road and Rail Transportation (S. 822-831). Boca Raton: CRC Press.

Meyer-Soylu, S., Parodi, O., Trenks, H., \& Seebacher, A. (2016). Das Reallabor als Partizipationskontinuum. Erfahrungen aus dem Quartier Zukunft und Reallabor 131 in Karlsruhe. Technikfolgenabschätzung - Theorie und Praxis, 25 (3), (S. 31-40).

Mobilität in Deutschland (MID) (2008). Bundesministerium für Verkehr und digitale Infrastruktur, Berlin. http://daten.clearingstelle-verkehr.de/223/. Zugegriffen am 02.03.2019.

Müller, J., Renyi, M., \& Kunze, C. (2014). Partizipative Technikentwicklung für ein selbstbestimmtes Leben im Alter - ein Exkurs. Participartory technology development for an independent life for senior citizens. Transdisziplinäre Konferenz zum Thema „Technische Unterstützungssysteme, die die Menschen wirklich wollen“, Hamburg (15.-16.12.2014). https://www.researchgate.net/publication/269703236_Partizipative_ Technikentwicklung fur_ein_selbstbestimmtes_Leben_im_Alter_- ein_Exkurs Participartory_technology_development_for_an_independent_life_for_senior_citizens. Zugegriffen am 04.03.2019. 
Plattner, H., Meinel, C., \& Weinberg, U. (2009). Design Thinking - Innovation lernen Ideenwelten öffnen. München: mi-Wirtschaftsbuch.

Pruitt, J., \& Adlin, T. (2006). The persona lifecycle: Keeping people in mind throughout product design. San Francisco: Morgan Kaufmann Publishers.

Quint, A., Alcántara, S., \& Seebacher, A. (2018). Der Partizipationsmythos „Partizipation in Reallaboren ist per se transparent und muss es auch sein“. In R. Defila \& A. Di Giulio (Hrsg.), Transdisziplinär und transformativ forschen. Eine Methodensammlung (S. 69-73). Wiesbaden: Spinger VS. doi: 10.1007/978-3-658-21530-9_3.

Røe, P. G. (2000). Qualitative research on intra-urban travel: an alternative approach. Journal of Transport Geography, 8, (S. 99-106). doi: 10.1016/S0966-6923(99) 00039-3.

Sanders, E. B. N. (2003). From user-centered to participatory design approaches. In J. Frascara (Hrsg.), Design and the social sciences (S. 18-25). Abingdon: Taylor \& Francis Books Limited.

Scheiner, J. (1998). Aktionsraumforschung auf phänomenologischer und handlungstheoretischer Grundlage. Geographische Zeitschrift, 86 (1), (S. 50-66).

Seebacher, A., Alcántara, S., \& Quint, A. (2018). Der Partizipationsmythos „Partizipation bedeutet, alle immer an allem zu beteiligen“. In R. Defila \& A. Di Giulio (Hrsg.), Transdisziplinär und transformativ forschen. Eine Methodensammlung (S. 101-104). Wiesbaden: Spinger VS. doi: 10.1007/978-3-658-21530-9_5.

Selle, K. (2013). Über Bürgerbeteiligung hinaus: Stadtentwicklung als Gemeinschaftsaufgabe? Analysen und Konzepte. Detmold: Verlag Dorothea Rohn.

Van Someren, M. W., Barnard, Y. F., \& Sandberg, J. A. C. (1994). The Think Aloud Method - A Practical Guide to Modelling Cognitive Processes. Amsterdam, Boston: Academic Press.

Verband Region Stuttgart (2011). Begleituntersuchungen zur Fortschreibung des Regionalverkehrsplans - Band 1: Mobilität und Verkehr in der Region Stuttgart 2009/2010. Regionale Haushaltsbefragung zum Verkehrsverhalten. https://www.region-stuttgart. org/information-und-download/veroeffentlichungen/schriftenreihe/. Zugegriffen am 08.03.2019. 


\section{Anhang}

Anlage a: Leitfaden für die Expertengespräche im Reallabor Schorndorf - (am Beispiel eines Interviews mit einem Vertreter des Seniorenforums):

Frage 1: Besteht bei Senior(inn)en ein Bedarf für eine bessere Mobilität?

Frage 2: Welche Vor- bzw. Nachteile werden durch einen bedarfsgerechten ÖPNV erwartet?

Frage 3: Ist die Anforderung eines Busses via App für Senior(inn)en realistisch bzw. welche anderen Lösungsansätze sind vorstellbar? Wie könnte ein einfaches Bedienkonzept aussehen?

Frage 4: Inwieweit ist zu erwarten, dass die Senior(inn)en Schorndorfs aufgeschlossen gegenüber Neuerungen/neuen Konzepten sind?

Frage 5: Welche Anforderungen bestehen an die neuen Busse? Bspw. in Form von Fahrrad- und Rollstuhlmitnahme.

Frage 6: Welche Einsatzzwecke bzw. -gebiete sehen Sie für ein solches Bussystem in Bezug auf die Bedürfnisse der Senior(inn)en Schorndorfs?

Frage 7: Wie bzw. wo sollte solch ein System bei Senior(inn)en beworben werden? 
Anlage b: Fragebogen für Feedback nach dem Co-Creation-Workshop I

Wir bitten Sie, die folgenden Fragen möglichst vollständig zu beantworten. Die Erhebung dient ausschließlich wissenschaftlichen Zwecken. Die Auswertung erfolgt anonym.

Wie häufig benutzen Sie die folgenden Verkehrsmittel? (bitte ankreuzen, Mehrfachnennungen möglich)

\begin{tabular}{|l|c|c|c|c|}
\hline & $\begin{array}{c}\text { Täglich/ } \\
\text { fast täglich }\end{array}$ & $\begin{array}{c}\text { Häufig/ } \\
\text { mind. 1x } \\
\text { pro Woche }\end{array}$ & $\begin{array}{c}\text { Selten/ } \\
\text { ca. 1x pro } \\
\text { Monat }\end{array}$ & $\begin{array}{c}\text { Nie bzw. } \\
\text { fast nie }\end{array}$ \\
\hline Pkw, als Fahrer(in) & $\square$ & $\square$ & $\square$ & $\square$ \\
\hline Pkw, als Mitfahrer(in) & $\square$ & $\square$ & $\square$ & $\square$ \\
\hline Carsharing-Pkw & $\square$ & $\square$ & $\square$ & $\square$ \\
\hline $\begin{array}{l}\text { Öffentliche Verkehrsmittel } \\
\text { (Bus, Bahn, etc.) }\end{array}$ & $\square$ & $\square$ & $\square$ & $\square$ \\
\hline $\begin{array}{l}\text { Motorrad, Roller, Mofa, } \\
\text { o. ä. }\end{array}$ & $\square$ & $\square$ & $\square$ & $\square$ \\
\hline Fahrrad & $\square$ & $\square$ & $\square$ & $\square$ \\
\hline $\begin{array}{l}\text { Zu Fuß (ohne Wege zum/ } \\
\text { vom Auto oder S-Bahn etc.) }\end{array}$ & $\square$ & $\square$ & $\square$ & $\square$ \\
\hline anderes Verkehrsmittel: & $\square$ & $\square$ & $\square$ & $\square$ \\
\hline
\end{tabular}

Welches Verkehrsmittel benutzen Sie am liebsten, welches am häufigsten? (bitte ankreuzen)

\begin{tabular}{|l|c|c|}
\hline & ...am liebsten & ...am häufigsten \\
\hline Pkw & $\square$ & $\square$ \\
\hline Öffentliche Verkehrsmittel (Bus, Bahn, etc.) & $\square$ & $\square$ \\
\hline Motorrad, Roller, Mofa, o. ä. & $\square$ & $\square$ \\
\hline Fahrrad & $\square$ & $\square$ \\
\hline Zu Fuß & $\square$ & $\square$ \\
\hline anderes Verkehrsmittel: & $\square$ & $\square$ \\
\hline
\end{tabular}




\begin{tabular}{|c|c|c|c|c|c|c|c|}
\hline \multicolumn{4}{|c|}{$\begin{array}{l}\text { Besitzen Sie oder andere Personen in } \\
\text { Ihrem Haushalt ein eigenes Auto? (bitte } \\
\text { ankreuzen) }\end{array}$} & \multicolumn{4}{|c|}{$\begin{array}{l}\text { Teilen Sie sich das Auto mit anderen } \\
\text { Personen? (bitte ankreuzen) }\end{array}$} \\
\hline$\square$ & $\mathrm{Ja}$ & $\square$ & Nein & $\square$ & $\mathrm{Ja}$ & $\square$ & Nein \\
\hline
\end{tabular}

Angaben zur Person:

\begin{tabular}{|c|c|c|c|c|c|c|c|c|c|c|c|}
\hline \multicolumn{7}{|l|}{ Wohnort (Teilort) } & & & & & \\
\hline \multicolumn{12}{|l|}{ Alter } \\
\hline \multicolumn{12}{|l|}{ Geschlecht } \\
\hline \multicolumn{12}{|l|}{ Höchster Bildungsabschluss } \\
\hline \multicolumn{12}{|c|}{ Anzahl der im Haushalt lebenden Kinder (unter 18) } \\
\hline \multirow[t]{2}{*}{ Derzeitiger Erwerbsstatus } & \multicolumn{2}{|c|}{ 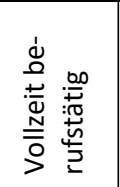 } & \multicolumn{2}{|c|}{ 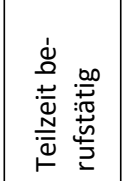 } & 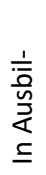 & \multicolumn{3}{|c|}{ 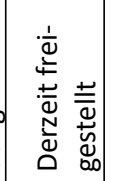 } & 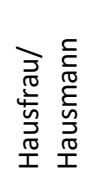 & 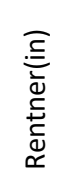 & $\begin{array}{l}\frac{n}{0} \\
\frac{n}{d} \\
\frac{0}{2} \\
\frac{0}{2}\end{array}$ \\
\hline & $\square$ & & ᄃ & & $\square$ & & $\square$ & & $\square$ & ㅁ & $\square$ \\
\hline \multirow[t]{2}{*}{$\begin{array}{l}\text { Monatliches Nettoeinkom- } \\
\text { men (gesamtes Einkommen } \\
\text { nach Abzug von Steuern } \\
\text { und Sozialversicherungsbei- } \\
\text { trägen) }\end{array}$} & $\begin{array}{c}\text { Un- } \\
\text { ter } \\
500 €\end{array}$ & $\begin{array}{r}50 \\
b \\
\text { un } \\
1.0 \\
€\end{array}$ & & $\begin{array}{r}1.0 c \\
€ b \\
\text { unt } \\
2.00 \\
€\end{array}$ & & $\begin{array}{l}2.00 \\
€ \text { bi } \\
\text { unte } \\
3.00 \\
€\end{array}$ & & $\begin{array}{l}3.000 \\
€ \text { bis } \\
\text { unter } \\
4.000 \\
€\end{array}$ & & & $\begin{array}{c}\text { Über } \\
6.000 €\end{array}$ \\
\hline & $\square$ & c & & $\square$ & & $\square$ & & $\square$ & & & $\square$ \\
\hline
\end{tabular}


1. Welche Erwartungen hatten Sie an den Workshop?

2. Was hat Ihnen an diesem heutigen Tag besonders gut gefallen?

3. Was hat Ihnen an diesem heutigen Tag überhaupt nicht gefallen?

4. Was möchten Sie uns noch mitteilen, was heute nicht thematisiert wurde?

5. Konnten Sie sich mit dem Nutzerprofil und dem Szenario der Busnutzung identifizieren?

6. Wären Sie grundsätzlich bereit, auch am zweiten Workshop teilzunehmen?

$\square$ Ja, ich möchte gerne teilnehmen $\quad \square$ Nein, ich habe kein Interesse

Vielen Dank für Ihre Teilnahme! 
Open Access Dieses Kapitel wird unter der Creative Commons Namensnennung 4.0 International Lizenz (http://creativecommons.org/licenses/by/4.0/deed.de) veröffentlicht, welche die Nutzung, Vervielfältigung, Bearbeitung, Verbreitung und Wiedergabe in jeglichem Medium und Format erlaubt, sofern Sie den/die ursprünglichen Autor(en) und die Quelle ordnungsgemäß nennen, einen Link zur Creative Commons Lizenz beifügen und angeben, ob Änderungen vorgenommen wurden.

Die in diesem Kapitel enthaltenen Bilder und sonstiges Drittmaterial unterliegen ebenfalls der genannten Creative Commons Lizenz, sofern sich aus der Abbildungslegende nichts anderes ergibt. Sofern das betreffende Material nicht unter der genannten Creative Commons Lizenz steht und die betreffende Handlung nicht nach gesetzlichen Vorschriften erlaubt ist, ist für die oben aufgeführten Weiterverwendungen des Materials die Einwilligung des jeweiligen Rechteinhabers einzuholen.

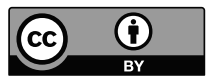

